\title{
Are ecological communities the seat of endosymbiont horizontal transfer and diversification? A case study with soil arthropod community
}

\author{
Manisha Gupta $^{1}$ | Rajbir Kaur ${ }^{1,2}$ | Ankita Gupta ${ }^{3}$ | Rhitoban Raychoudhury ${ }^{1} \mathbb{C}$
}

${ }^{1}$ Indian Institute of Science Education and Research, Mohali (IISER-Mohali), Manauli, India

${ }^{2}$ Indian Institute of Science, Bengaluru, India ${ }^{3}$ ICAR- National Bureau of Agricultural Insect Resources (NBAIR), Bengaluru, India

\section{Correspondence}

Rhitoban Raychoudhury, Indian Institute of Science Education and Research, Mohali (IISER-Mohali), Knowledge city, Sector 81, SAS Nagar, Manauli, PO 140306, Punjab, India.

Email: rhitoban@iisermohali.ac.in

\section{Funding information}

This work was supported by the funds from the Indian Institute of Science Education and Research (IISER) Mohali. MG was supported by a fellowship from University Grant commission (UGC).

\begin{abstract}
Maternally inherited endosymbionts of arthropods are one of the most abundant and diverse group of bacteria. These bacterial endosymbionts also show extensive horizontal transfer to taxonomically unrelated hosts and widespread recombination in their genomes. Such horizontal transfers can be enhanced when different arthropod hosts come in contact like in an ecological community. Higher rates of horizontal transfer can also increase the probability of recombination between endosymbionts, as they now share the same host cytoplasm. However, reports of community-wide endosymbiont data are rare as most studies choose few host taxa and specific ecological interactions among the hosts. To better understand endosymbiont spread within host populations, we investigated the incidence, diversity, extent of horizontal transfer, and recombination of three endosymbionts (Wolbachia, Cardinium, and Arsenophonus) in a specific soil arthropod community. Wolbachia strains were characterized with MLST genes whereas 16 S rRNA gene was used for Cardinium and Arsenophonus. Among 3,509 individual host arthropods, belonging to 390 morphospecies, $12.05 \%$ were infected with Wolbachia, $2.82 \%$ with Cardinium and $2.05 \%$ with Arsenophonus. Phylogenetic incongruence between host and endosymbiont indicated extensive horizontal transfer of endosymbionts within this community. Three cases of recombination between Wolbachia supergroups and eight incidences of withinsupergroup recombination were also found. Statistical tests of similarity indicated supergroup A Wolbachia and Cardinium show a pattern consistent with extensive horizontal transfer within the community but not for supergroup B Wolbachia and Arsenophonus. We highlight the importance of extensive community-wide studies for a better understanding of the spread of endosymbionts across global arthropod communities.
\end{abstract}

KEYWORDS

DNA barcoding, ecological community, endosymbionts, horizontal transfer, recombination 


\section{1 | INTRODUCTION}

Maternally inherited endosymbionts, infecting arthropods, are one of the most diverse and abundant of all bacteria infecting them. About two-third of all terrestrial arthropods are infected with at least one maternally inherited endosymbiont (Hilgenboecker et al., 2008) and can play crucial roles in the ecology and evolution of their hosts (Gebiola et al., 2017; Semiatizki et al., 2020). The most common of these endosymbionts are Wolbachia, Cardinium, Arsenophonus, Rickettsia and Spiroplama. Out of these, Wolbachia remains the most widely distributed with an incidence rate of $16-66 \%$ (Hilgenboecker et al., 2008; Weinert et al., 2015; Werren, Windsor, et al., 1995) and comprising of 18 different clades (supergroups $A$ to R) (Landmann, 2019). Incidence of the other endosymbionts varies from 4-10\% (Duron et al., 2008; Zchori-Fein \& Perlman, 2004).

An important evolutionary feature of these endosymbionts is the lack of phylogenetic congruence with their hosts (Dewayne Shoemaker et al., 2002; Werren, et al., 1995). This indicates that although they undergo vertical transmission in the short term, over the course of their evolutionary histories, they have also undergone extensive horizontal transfer across taxonomically unrelated hosts (Werren et al., 2008). This is also evident from the occurrence of similar endosymbiont strains in taxonomically unrelated hosts and conversely, the presence of divergent strains in closely related hosts (Vavre et al., 1999). Individual arthropods can harbor a single or multiple strain of any one endosymbiont as well as multiple strains of different endosymbionts (Zhang et al., 2016). The presence of more than one type of endosymbiont in a single host can be indicative of the utilization of the same host by different endosymbionts to spread across different arthropod communities (Russell et al., 2012; Zélé et al., 2018; Zhao et al., 2013).

Another key feature of endosymbionts is the pervasive recombination seen in their genomes (Ellegaard et al., 2013). This has been particularly well documented in Wolbachia (Malloch \& Fenton, 2005) as well as in other endosymbionts (Mouton et al., 2012). This level of recombination is extensive enough to render single-gene phylogenies unable to properly reflect the evolutionary history of an endosymbiont strain and, therefore, has necessitated the development of multilocus strain typing (MLST) systems (Maiden et al., 1998). Such MLST studies (Hou et al., 2020), as well as whole-genome analysis of endosymbionts (Wang et al., 2020), indicate extensive recombination within them. However, for recombination to happen, at least two endosymbiont strains must be present in the same cytoplasm of a particular host. This is possible through horizontal transfer of endosymbionts to different hosts. During this process, different endosymbionts can find themselves sharing the same cytoplasm with resident endosymbionts and can also lead to multiple infections. The rate at which this leads to stable multiple infections is not known, but this obviously creates opportunities for genetic exchange between endosymbionts. Moreover, such coinfections can trigger selection whereby only a single endosymbiont can remain within a host. Such flux seems to be a key feature of endosymbiont dynamics, especially with Wolbachia, where loss is 1.5 times higher than acquisition of new infections (Bailly-Bechet et al., 2017). Additionally, this cooccurrence can lead to increased chances of recombination between these strains especially if the new host is already infected with other strains. Evidence for such recombination is also well documented. The presence of a very similar recombinant Wolbachia strains has been observed in the parasitoid wasp Nasonia and its host Protocalliphora (Werren \& Bartos, 2001), as well as in Anastrepha fruit flies and their parasitoid braconid wasps (Mascarenhas et al., 2016).

Horizontal transfer, therefore, can not only increase the taxonomic range of hosts that a particular endosymbiont can infect, but can also be responsible for increased incidences of recombination between endosymbionts. Moreover, horizontal transfer of endosymbionts can increase if different hosts, with different resident infections, come in contact like in host-parasite, host-parasitoid, prey-predator, and other ecological interactions (Sanaei et al., 2021). Examples where host-parasitoid interactions have been implicated for such transfer include the presence of similar Wolbachia strains among frugivorous Drosophila and their hymenopteran parasitoid (Vavre et al., 1999), Nasonia vitripennis and Muscidifurax uniraptor sharing similar Wolbachia with their fly host Protocalliphora (Baudry et al., 2003) and transmission of Wolbachia into whitefly via parasitoid wasps (Ahmed et al., 2016). Another such ecological association which can lead to endosymbiont transfer is prey-predator interactions such as the predatory mite Metaseiulus occidentalis and its prey Tetranychus urticae (spider mite) sharing similar endosymbionts (Hoy $\&$ Jeyaprakash, 2005). Some parasitic mites, such as Tyrophagus putrescentiae, can also facilitate the transfer of Wolbachia to different Drosophila host populations (Brown \& Lloyd, 2015). Similarly, horizontal transfer of endosymbionts can also be host plant mediated as observed in the transfer of Cardinium to different leafhopper species (Gonella et al., 2015) as well as horizontal transfer of Wolbachia in whitefly through cotton leaves (Li et al., 2017). Shared food resources between hosts can also mediate horizontal transfer of endosymbionts (Tolley et al., 2019).

It is clear from these examples that horizontal transfer of endosymbionts is possibly taking place when two hosts are coming together to perform a particular ecological interaction and thus have overlapping niches (Sanaei et al., 2021). The endosymbiont present within these hosts can then be serendipitously transferred from one host to the other. Therefore, to understand the dynamics of the spread of endosymbionts through horizontal transfer, one needs to look at the level where most of these ecological associations are taking place, which could be within the boundaries of ecological community. A well-defined ecological community will have several diverse host taxa with significant overlap of niches as they are interacting with each other. This physical contact of the hosts can facilitate the horizontal transfer of their resident endosymbionts (Sanaei et al., 2021). Moreover, many host taxa can belong to many different ecological communities (Morrow et al., 2014) and this cosmopolitan nature of a few host taxa will further facilitate the spread of endosymbionts from one ecological community to another, almost like spreading through a metacommunity (Brown et al., 2020). Therefore, investigating endosymbiont diversity and 
horizontal transfer within specific ecological communities seems logical. Yet, there are very few studies that have taken this approach and instead focus mainly on endosymbiont spread within a particular habitat (Stahlhut et al., 2010) or in a specific genus (Baldo et al., 2008; Raychoudhury et al., 2009; Turelli et al., 2018) or within specific host taxa (Ahmed et al., 2016; Tolley et al., 2019). Among community-wide surveys, Kittayapong et al. (2003) demonstrated Wolbachia strain diversity within rice field arthropod community, whereas Sintupachee et al. (2006) reported plant-mediated horizontal transfer among arthropod community found on pumpkin leaves. However, to understand whether such horizontal transfer can be correlated with relatively higher rates of recombination is difficult as most of these studies are based on single-gene phylogenies. Another crucial effect of this view of within-community horizontal transfer of endosymbionts can lead to an important hypothesis about sequence diversity of the endosymbionts themselves. If endosymbionts are rapidly undergoing horizontal transfer within a particular ecological community, then very similar bacterial strains would be found among the arthropod hosts of that community. This would make these bacteria more closely related to each other, resulting in lower than expected pairwise sequence divergence among them. This can then serve as a signature of recent and relatively rapid communitywide horizontal transfer of resident endosymbionts.

In the present study, we test whether such horizontal transfer and resulting recombination are happening within the endosymbionts of a diverse soil arthropod community. Three major endosymbionts, Wolbachia, Cardinium, and Arsenophonus, were selected and screened across arthropod hosts. We investigated Wolbachia sequence diversity using the well-established MLST scheme (Baldo et al., 2006) and also identified specific recombination events. We also investigated Cardinium and Arsenophonus incidence with $16 \mathrm{~S}$ rRNA gene sequences. A statistical model was then used to test whether the endosymbiont found within this community is more closely related to each other than expected. Our study indicates that supergroup A Wolbachia and Cardinium are indeed showing greater sequence similarity within the community indicating, perhaps, that such horizontal transfer events are more prevalent in these two endosymbionts than in supergroup B Wolbachia and Arsenophonus.

\section{2 | MATERIALS AND METHODS}

\subsection{Sample collection and morphological identification of soil arthropods}

To access soil arthropod biodiversity, sampling was done in October and November 2015 (a postmonsoon season) from a relatively undisturbed land $\left(220 \times 70 \mathrm{~m}^{2}\right)$ near the vicinity of the host institution $\left(30^{\circ} 39^{\prime} \mathrm{N}\right.$ $76^{\circ} 43^{\prime} \mathrm{E}$, Mohali, Punjab, India; Figure S1A). The study area was naturally divided into roughly eight quadrants by plantations of poplar (Figure S1B,C). Five randomly selected quadrants were sampled by collecting leaf litter and pitfall traps. These two independent sampling methods were used in tandem to give a more comprehensive sampling of the resident species (Olson, 1991; Querner \& Bruckner, 2010) as pitfall traps are biased toward surface-active taxa, whereas leaf litter method is biased toward less active taxa. Two parallel transects, each $30 \mathrm{~m}$ long, were marked across each quadrant using a rope (Figure S1B,D). Each of these transects was marked at $10 \mathrm{~m}$ intervals, and two alternatively marked points were sampled for leaf litter, while pitfall traps were placed at the other two ends (Figure S1E). Samples from each type of collections were later combined. In total, 20 collections, each from leaf litter and pitfalls, were obtained.

Leaf litter was collected from an area of roughly $0.09 \mathrm{~m}^{2}$ (Figure S1F) and immediately placed in a plastic bag. Additionally, each leaf litter sample was accompanied by a soil sample of an area $282 \mathrm{~cm}^{3}$ immediately below it (Sabu \& Shiju, 2010). Samples were weighed so that each sample roughly had the same weight (500$600 \mathrm{gm})$ and were then settled in a series of Tullgren funnels with a $100 \mathrm{~W}$ light. The emerging arthropods were collected in a $50 \mathrm{ml}$ beaker, with absolute ethanol, continuously for the next 4-6 days or until no arthropod samples emerged. Emerged arthropods were collected every $24 \mathrm{hr}$, and $100 \mathrm{ml}$ of fresh absolute ethanol was added to the collection beaker. The pitfall traps were settled on the ground by placing a $250-\mathrm{ml}$ beakers with $50 \mathrm{ml}$ of absolute ethanol (Figure S1G). Samples were collected every other day with a replacement of fresh ethanol.

Arthropods obtained from each of pitfall and leaf litter samples (20 samples each) were sorted individually with multiple washes in fresh ethanol to reduce cross-contamination. Detailed (dorsal and lateral) views of each individual arthropod obtained were photographed under a stereomicroscope (M205C, Leica Microsystems) with scale ranging from 0.2 to $2 \mathrm{~mm}$. These were then sorted according to their morphology into morphospecies and provisionally identified till order level. To evaluate whether the sampling method employed yielded a significant proportion of the community diversity, several diversity indices were calculated with EstimateS v9.1.0 (Colwell, 2013) such as ACE (Abundance coverage estimator; Chao et al., 2000), Chao1 (Chao, 1984), ICE (Incidence coverage estimator), Chao2, and Jack 1 and Jack 2 (Smith \& van Belle, 1984).

Cross-contamination of both host and endosymbiont DNA is one of the possible caveats (Zettler et al., 2020), of collecting arthropod samples from the environment in bulk and subsequent storage. Although, previous reports indicate that this is unlikely (Duplouy et al., 2009), yet to minimize this, the collected and sorted morphospecies were washed in ethanol multiple times.

For morphospecies which had more than three individuals, DNA was extracted from a single individual by either the $\mathrm{HiPurA}^{\mathrm{TM}}$ insect DNA purification kit (HIMEDIA) or by using the phenol-chloroformisoamyl alcohol (PCI) method. In $\mathrm{PCl}$ method, samples were crushed in $200 \mu \mathrm{l}$ lysis buffer containing $10 \mathrm{mM}$ each of Tris- $\mathrm{HCl}(\mathrm{pH}$ 8.0), EDTA ( $\mathrm{pH}$ 8.0) and $\mathrm{NaCl}$. DNA was precipitated using isopropanol and dissolved in $1 \mathrm{XTE}$ (pH 8.0). For morphospecies which only had single individuals, a different nondestructive extraction protocol was used (Rowley et al., 2007). Whole individuals were incubated at $60^{\circ} \mathrm{C}$ in $100-400 \mu \mathrm{l}$ of guanidinium thiocyanate (GuSCN) based extraction buffer (GuSCN, 0.1M Tris- $\mathrm{HCl}, 0.2 \mathrm{M}$ EDTA with Triton 
$\mathrm{X}-100)$ for 1-4 hr. The individuals were then removed for storage, and the DNA remaining in the buffer was precipitated using isopropanol. Extracted DNA was quantified using the NanoDrop ${ }^{\text {TM }} 2000$ spectrophotometer (Thermo Fisher Scientific), and PCR suitability was accessed by running a PCR using $28 S$ primers (Table 1 ).

The morphospecies were barcoded (Hebert et al., 2003) using the $\sim 600 \mathrm{bp}$ of the mitochondrial CO1 gene (Table 1). 2-20 ng/ $\mathrm{l}$ of extracted DNA was used in $20 \mu \mathrm{l}$ PCRs with an initial denaturation step at $95^{\circ} \mathrm{C}$ for $3 \mathrm{~min}, 39 \mathrm{cycles}$ of denaturation $\left(95^{\circ} \mathrm{C}, 45 \mathrm{~s}\right)$, annealing $\left(51-56^{\circ} \mathrm{C}\right.$ for $\left.45 \mathrm{~s}\right)$, extension $\left(72^{\circ} \mathrm{C}, 1 \mathrm{~min}\right)$, and a final extension at $72^{\circ} \mathrm{C}$ for $10 \mathrm{~min}$. PCR products were visualized on $1 \%$ agarose gels and then cleaned with Exonuclease I and Shrimp alkaline Phosphatase (New England Biolabs Inc.) before being sequenced with the BigDye ${ }^{\circledR}$ Terminator v3.1 Cycle Sequencing Kit. Initially, only the forward strand was sequenced and if any base ambiguity was observed, then the reverse strand was also sequenced. This was especially done to detect any cases where due to prey-predator, host-parasite, host-parasitoid, and other ecological interactions, multiple arthropod hosts could have been present in any one sample. These hosts would then yield multiple peaks in the $\mathrm{CO} 1$ chromatograms as DNA from more than one host would be coamplified. However, we did not find any such discrepancy in our dataset.

CO1 sequences obtained were identified through the NCBI (Johnson et al., 2008) and BOLD databases (Ratnasingham \& Hebert, 2007) by BLAST (last performed in August 2019). The "best hit" obtained was used to check the provisionally identified morphospecies. If both databases yielded the same hit, then it was determined to have been identified. If they yielded different hits, then the taxonomic identification was moved down to the level common between these two hits. These results were further cross-checked with the photographic data to establish whether the $\mathrm{CO} 1$ sequences also matched up with the provisional taxonomic identity of the morphospecies. Only unique morphospecies were included in further analysis after analyzing the CO1 sequences (Table S2).

\section{2 | Endosymbiont screening and phylogenetic analysis}

All the 390 morphospecies obtained were screened for the three endosymbionts-Wolbachia, Cardinium, and Arsenophonus. Incidence of each of these endosymbionts was estimated using primers specific to them (Table 1). The multilocus strain typing (MLST) system was used (Baldo et al., 2006) to identify and characterize the Wolbachia infections. For Cardinium and Arsenophonus, $16 \mathrm{~S}$ rRNA gene was amplified using specific primers (Table 1). Nasonia vitripennis infected with both $A$ and $B$ supergroups of Wolbachia was used as positive control for Wolbachia screening, and Bemisia tabaci strains with Cardinium and Arsenophonus infections were used for screening the other two endosymbionts. Autoclaved distilled water was used as no-template negative controls.

To test for the presence of Wolbachia, the wspec primers were used (Table 1). Samples positive for wspec were then sequenced for one of the MLST genes, usually fbpA, to identify single Wolbachia infections by inspecting the chromatograms for multiple peaks. Samples with multiple Wolbachia infection were not processed further as assigning a particular Wolbachia MLST allele to a particular strain would have been impossible. The MLST allele sequences were compared with existing sequences in PubMLST database (Jolley et al., 2018) to identify their allele profiles (unique numbers assigned for each unique sequence) and sequence type (ST) which is a new strain type as defined by the combination of five MLST allele profiles (Baldo et al., 2006). Unique sequences were submitted to the PubMLST database for curation. Sequences obtained from this study were also deposited in NCBI and BOLD databases (Table S2).

Sequences were aligned with Sequencher 5.2.4 (Gene Codes Corporation) and manually edited with BioEdit v. 7.2.5 (Hall, 1999). DNA sequence evolution models were computed using MEGA7 (Kumar et al., 2016). GTR+g (general time-reversible model with $\gamma$-distributed rate variation) was found to be the best model for all CO1 phylogenetic trees. The appropriate models found for other datasets were as follows: K2+g (Kimura's 2-parameter model) for both Cardinium and Arsenophonus; T92+g+i (Tamura's 3-parameter model with $\gamma$-distributed rate variation and proportion of invariable sites) for concatenated MLST dataset; T92+g for gatB, hcpA, ftsZ, fbpA gene; HKY+g (Hasegawa, Kishino, and Yano's model) for coxA. Bayesian phylogeny was constructed for $\mathrm{CO} 1$ sequences using MrBayes v3.2.5 (Ronquist et al., 2012). Each phylogenetic analysis was run at least twice and was accepted only if there was no change in the major branching order (Figure S2). Maximum-likelihood phylogenetic trees of Wolbachia, Cardinium, and Arsenophonus were constructed in MEGA7 with 1,000 bootstrap replicates for each. Phylogenetic trees were visualized and edited with Figtree v1.4.2 (Rambaut, 2009).

To account for the frequent recombination seen in Wolbachia genomes, ClonalFrame v2.1 (Didelot \& Falush, 2007) was used to infer phylogeny from MLST data. ClonalFrame was run for $3 \times 10^{5}$ iterations with the first $50 \%$ iterations discarded as burn-ins. Estimates of recombination rate were also obtained.

\section{3 | Identifying horizontal transfers, recombination events, and test of endosymbiont similarity}

To test for horizontal transmission of endosymbionts across the soil arthropod hosts, three sets of analyses were done. The first was a qualitative estimation of the horizontal transfer obtained by comparing host and endosymbiont phylogenies. The second was a quantitative estimation of the correlation between the pairwise distance matrices of hosts and their corresponding endosymbionts by the Spearman method ( $r$ ) of Mantel test for correlation (Legendre \& Legendre, 2012) which was done in R v1.2.5. A total of five different correlations were computed with their corresponding host sequences: all the Wolbachia-infected samples (33), only A supergroup Wolbachia-infected samples (16), only B supergroup Wolbachia-infected samples (15), only Cardinium-infected samples 




总总


骂
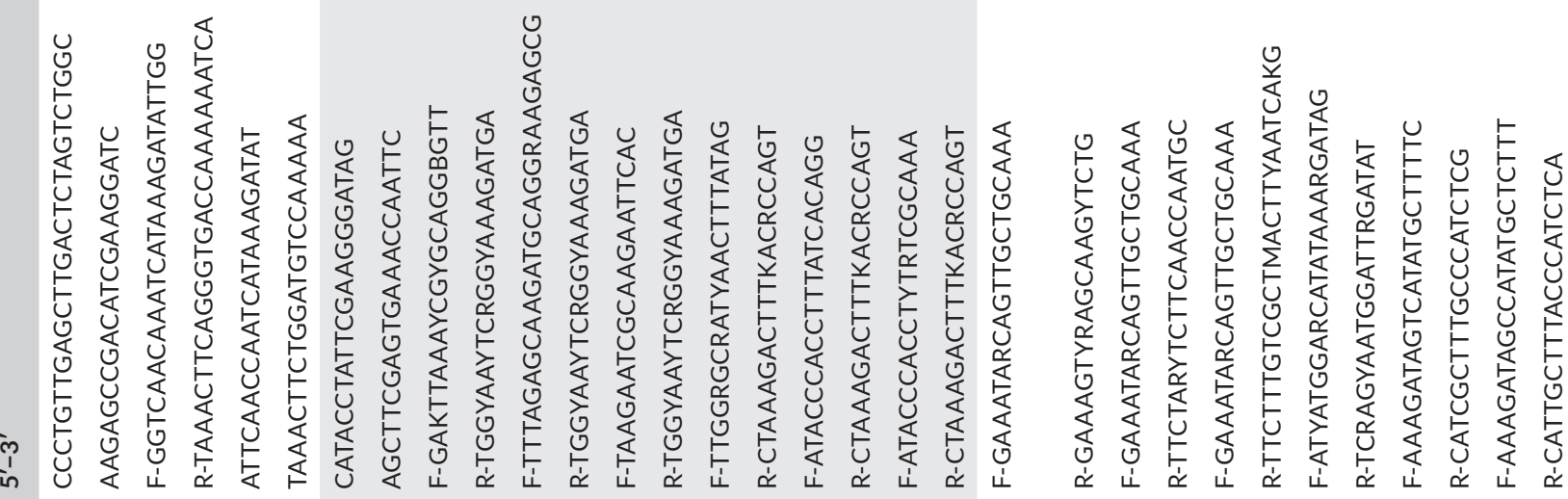

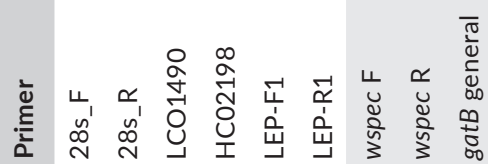

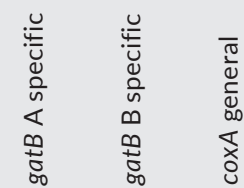



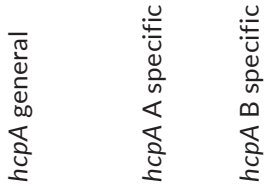

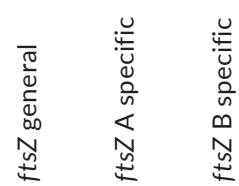



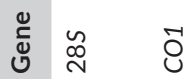

\begin{tabular}{ll}
0 & 0 \\
\multirow{0}{*}{} & $\stackrel{0}{0}$
\end{tabular}

홍

畐

N

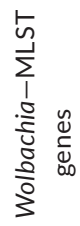


(eight), and Arsenophonus-infected samples (seven). In addition, ParaFit (Legendre et al., 2002) and PACo (Procrustean Approach to Cophylogeny) programs (Balbuena et al., 2013) were used to test for association between host and endosymbiont pairwise distances.

To identify and visualize recombination events within the Wolbachia concatenated MLST sequences (2079 bp), SplitsTree v4.14.8 (Huson et al., 2008) was used to construct phylogenetic network using uncorrected $p$-distance and neighbor-net method (Bryant \& Moulton, 2004). To statistically evaluate presence of recombination, $\Phi$ test (Bruen et al., 2006) was used in SplitsTree v4.14.8. These identified events were then further evaluated in RDP4 v4.97 (Martin et al., 2015). This program has been used to detect recombination events in similar studies (Baldo, Bordenstein, et al., 2006; Hou et al., 2020; Yang et al., 2013) and uses a pairwise scanning approach to detect recombination within a group of aligned sequences (Martin et al., 2015; Martin \& Rybicki, 2000). It uses several suits of programs such as RDP (Martin \& Rybicki, 2000), GENECONV (Padidam et al., 1999), MaxChi (Smith, 1992), SiScan (Gibbs et al., 2000), BootScan (Martin et al., 2005), Chimera (Posada \& Crandall, 2001), and 3Seq (Boni et al., 2007) to detect and identify recombination events. A recombination event was considered for further analysis only when it was shown to be statistically significant by at least three or more of these detection methods. RDP was also used to detect the putative recombination breakpoints and their approximate positions in the concatenated dataset.

The model from Baldo et al. (2008) was used to test for the similarity of endosymbionts, where pairwise distance of endosymbiont strains found within the community was compared with strains available from different databases. To compare Wolbachia, MLST sequences, in the form of Wolbachia STs, were obtained from PubMLST database (last checked May, 2019). These were put in phylogenetic analyses with known supergroup sequences to identify whether they belonged to the A or the B supergroup. For Cardinium and Arsenophonus, homologues were obtained through NCBI Blast search and only those sequences were retained which had the same region of 165 rRNA amplified (last checked May, 2020). Mean pairwise distances were calculated in MEGA7 (Kumar et al., 2016) and then corrected with Jukes and Cantor model in DNAsp v5.10.01 (Librado \& Rozas, 2009). Mean pairwise distances were computed for endosymbionts within the community, their expected value obtained from the equivalent number of pairwise distances randomly selected from the database and all unique endosymbiont sequences obtained from PubMLST and NCBI databases. Pairwise distances were also computed separately for Wolbachia supergroup A, supergroup B, Cardinium, and Arsenophonus. The mean pairwise distance of endosymbionts presents in soil arthropod community was compared with an equivalent number of pairwise distances randomly selected from the database for 10,000 iterations. These iterations were computed to give a null distribution for comparison with the soil endosymbiont sequence data by Wilcox rank-sum test with continuity correction and $95 \%$ confidence interval (performed in $\mathrm{R}$ v1.2.5). Density plots for endosymbiont divergence from the soil arthropods and the databases were plotted in R (http://R-project.org). 


\section{3 | RESULTS}

\section{1 | Morphospecies diversity and endosymbiont infection frequencies}

A total of 3,509 individual arthropods were collected and sorted into 390 different morphospecies. Out of these, 198 morphospecies were exclusively obtained from the pitfall traps, 123 were obtained from leaf litter sampling, and 69 morphospecies were obtained from both. EstimateS provided diversity estimates ranging from $858( \pm 0)$, obtained from incidence coverage estimator, to $600( \pm 32.97)$ obtained through Jack 1 (Table S1). This indicates our sampling could capture $45-65 \%$ of the possible morphospecies in the community (Figure 1a). This is within expectations when compared to similar studies (Amancio et al., 2019; Rhoades et al., 2017).

These 390 morphospecies belonged to seven classes, 24 orders, 118 families, and 198 genera of arthropods (Table S2). We were able to amplify $\mathrm{CO} 1$ gene for 314 morphospecies. This was probably due to nucleic acid degradation as they were brought out of storage many times for visual identification, sorting, and photography and also underwent several washes in ethanol. Most of these samples were of single individuals (190 morphospecies) which prevented DNA extraction from additional samples.

Out of 390 morphospecies screened, 47 (12.05\%) were found to be infected with Wolbachia. Among these, $38.30 \%$ of them belonged to Hymenopterans, $25.53 \%$ to Hemiptera, $12.77 \%$ to Diptera, $8.51 \%$ each to Araneae and Coleoptera, $4.26 \%$ to Orthoptera, and $2.13 \%$ to Sarcoptiformes (Figure 1b). Two morphospecies, morph0081 and morph0085 (both Hymenoptera-Platygastridae), had multiple Wolbachia infections and therefore were not included for further analysis. There were nine infected morphospecies for which we were unable to amplify all the five MLST genes probably because of the above-mentioned DNA quality issues (Table S2). Since we screened only one individual per morphospecies, it is plausible that many infections were not detected as endosymbiont frequencies rarely reach fixation in host population. Therefore, the frequencies of endosymbionts in our study are a conservative estimate of actual infection rates.

We proceeded with the 36 unique host-Wolbachia combinations and 34 unique STs for which we could amplify all the five MLST genes. When resultant 180 allele profiles were compared with the existing sequences in PubMLST database, 77 new allelic profiles (14 each for gatB and coxA, 27 for hcpA, 12 for $\mathrm{ftsZ}$, and 10 for $\mathrm{fbpA}$ ) with 30 new STs were found (Table 2). For the strains labeled as ST-N1 and ST-N2, unique STs could not be assigned through PubMLST, as only one strand of gatB (ST-N1, ST-N2) and ftsZ (ST-N1) could be amplified (Table S2). As PubMLST requires chromatogram information from both strands, these were manually labeled as ST-N1 and ST-N2.

Phylogenetic analysis of the MLST data, using ClonalFrame, showed 17 Wolbachia strains from supergroup A, 15 from B supergroup and two strains from supergroup $F$ (Figure 2a). Supergroup A infections were predominantly found in Hymenoptera (70.5\%) whereas Hemipterans had mostly B supergroup infections (73.3\%).
Such host taxonomic bias of Wolbachia supergroups has been noted previously in Hymenopterans such as parasitoid wasps (Mohammed et al., 2017), ants (Russell et al., 2009), and bees (Gerth et al., 2013), in Hemipterans (Bing et al., 2014; Li et al., 2017), lepidopterans (Duplouy \& Hornett, 2018; Ilinsky \& Kosterin, 2017), and also in Dipterans (Stahlhut et al., 2010).

Eleven (2.82\%) of the morphospecies had Cardinium infections with four (33\%) each from Araneae and Hymenoptera, and one each from Entomobryomorpha, Mesostigmata, and Psocodea (Figure 2b). All the 11 Cardinium strains found in this study clustered with group A Cardinium (Nakamura et al., 2009). Three morphospecies, morph0085 (Hymenoptera-Platygastridae), morph0152 (Hymenoptera-Dicroscelio sp.), and morph0171 (HymenopteraTrichopria sp.) were found to be infected with both Wolbachia and Cardinium. Eight morphospecies (2.05\%) had Arsenophonus infections with two each from Hemiptera and Hymenoptera and one each from Diptera, Entomobryomorpha, Psocodea, and Thysanoptera (Figure 2c). Two morphospecies, morph0294 (HymenopteraPlatygastridae) and morph0329 (Hemiptera-Balclutha), were found to be infected with both Wolbachia and Arsenophonus. Morph0085 had multiple Wolbachia as well as Cardinium infections, whereas Morph0328 (Psocodea-Embidopsocus) had both Cardinium and Arsenophonus.

\section{2 | Horizontal transfer of endosymbiont strains}

The comparison of the phylogenies of host and their corresponding endosymbionts (Figure 3) suggests horizontal transfer of the endosymbionts within the soil arthropods. This was confirmed by a lack of significant correlations by Mantel test $(r)$ (entire Wolbachia dataset$r=0.205, p=0.002$; Wolbachia A supergroup $r=0.082, p=0.234$; B supergroup- $r=0.233, p=0.089$; Cardinium $-r=0.107, p=0.309$; Arsenophonus- $r=0.315, p=0.209$ ) between pairwise distances of host and their corresponding endosymbiont (Figure S5). However, PACo analysis showed some cophylogenetic pattern $(p<0.05)$ between host and their corresponding endosymbiont within all datasets (entire Wolbachia dataset- $\mathrm{m}^{2} \mathrm{xy}=0.288, p=0$; Wolbachia A supergroup $-\mathrm{m}^{2} \mathrm{xy}=0.49, p=0$; B supergroup $-\mathrm{m}^{2} \mathrm{xy}=0.52, p=0.04$; Cardinium- $\mathrm{m}^{2} \mathrm{xy}=0.19, p=0.01$; Arsenophonus- $\mathrm{m}^{2} \mathrm{xy}=0.17$, $p=0.04$; Figure S6), but ParaFit results suggest the same only for the entire Wolbachia dataset (ParaFit Global $=0.083, p=0.002$ ) and Wolbachia supergroup A (ParaFit Global $=0.001, p=0.032$ ). Evidence for cospeciation events was rejected (Table S3) for Wolbachia supergroup B (ParaFit Global $=0.001, p=0.19$ ), Cardinium (ParaFit Global $=9.67 \mathrm{E}-05, p=0.05$ ), and Arsenophonus (ParaFit Global $=6.97 \mathrm{E}-0.05, p=0.09$ ). Furthermore, it was not clear which host-endosymbiont links are contributing to overall congruency because PACo results differ from ParaFitLink1 and ParaFitLink2 (Table S3). The inconsistency between PACo and ParaFit analyses therefore indicates there is no unambiguous signal of codependence of host and endosymbiont phylogeny. However, if endosymbionts are first moving around the host taxa of this particular community, 
then very similar bacterial strains would be found in taxonomically distant soil arthropods. This is precisely what we found with two distinct Wolbachia strains. ST-541 and ST-559 were each found in two unrelated hosts (Table 2). Morph0001 (Orthoptera-Neonemobius) and morph0098 (Hemiptera-Phorodon) were both found to be infected with Wolbachia ST-541, whereas ST-559 was found in both morph0213 (Hemiptera-Heteropsylla) and morph0220 (HemipteraDelphacidae). Again, the possibility remains that these transfers could have happened independently and not correlated with the hosts being members of a particular community, but this assumes a nonparsimonious explanation that two independent events would converge on the transfer of the same Wolbachia ST in two different hosts.

Thus, horizontal transfer can also create opportunities where endosymbionts can potentially undergo recombination with each other since they are now in the same host cytoplasm.

\section{3 | Recombination events between endosymbiont strains}

Recombination in endosymbiont genomes is pervasive and can significantly increase diversification of these bacteria (Jiggins et al., 2001).

To check for incidence of recombination, we first analyzed the overall rates of recombination in the Wolbachia sequences with both ClonalFrame and RDP4. Both analyses showed a rate of nucleotide substitutions due to recombination/point mutation $(\mathrm{r} / \mathrm{m})$ of around 2.4 (95\% confidence interval between 1.4 and 3.7) which represents intermediate rates of recombination (Vos \& Didelot, 2009). This also indicates that recombination introduced twice more nucleotide substitutions, as compared to point mutations, in the Wolbachia datasets. Unsurprisingly, the $\Phi$ test in SplitsTree also showed significant evidence of recombination $(p<0.001)$ for the same Wolbachia sequences (Figure S3). However, for Cardinium and Arsenophonus, RDP4 did not indicate any evidence of recombination. This was probably due to the use of a single gene (16S rRNA gene) for these two bacteria.

To enumerate the recombination events within the Wolbachia sequences, we first looked at the phylogenetic trees to check whether single-gene phylogenies of all the five MLST genes (Figure S4) differ significantly with the concatenated MLST trees (Figure 4). Next, sliding-window algorithms in RDP4 were used to locate recombination breakpoints. The recombination events detected were then evaluated and confirmed manually. These analyses yielded several putative recombination events elaborated below.

\subsection{1 | Recombination between supergroups}

Three cases of acquisition of a gene or gene segments from different supergroups were detected. Phylogenetic and network analysis of concatenated MLST dataset (Figure 4) showed Wolbachia ST-N2, infecting morph0343 (Hymenoptera-Encyrtidae), to cluster with B supergroup, but individual gene trees revealed that the coxA fragment of ST-N2 clusters with A supergroup (Figure 4) and has the allelic profile of 7 . This phylogenetic disparity suggests that coxA gene of ST-N2 was acquired via recombination from a supergroup $A$ Wolbachia. Curiously enough, coxA allele 7 is also found in two other Wolbachia-infected hosts, ST-565 of morph0294 (HymenopteraPlatygastridae) and ST-544 of morph0076 (Araneae-Orthobula), both with supergroup A infections (Table 2). Although it is impossible to know which Wolbachia strains originally underwent recombination and gave rise to the recombinant allele 7 of $\operatorname{cox} A$, yet the presence of the same allele within the community suggests that the recombination event could have involved members within this ecological community. (a)

- ICE $(858 \pm 0)$ - Chao $2(743 \pm 65.35)$ - Jack $2(738 \pm 0)$

— ACE $(701 \pm 0)$ - Chao 1 (651 \pm 51.97$)$ — Jack $1(600 \pm 32.97)$

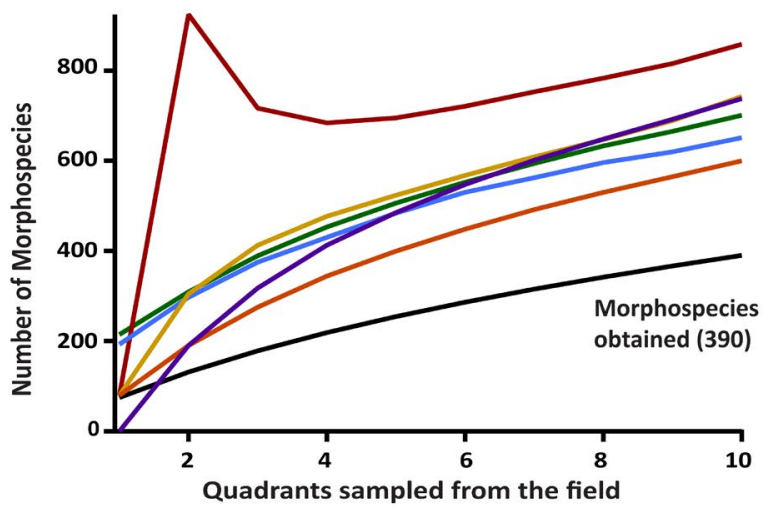

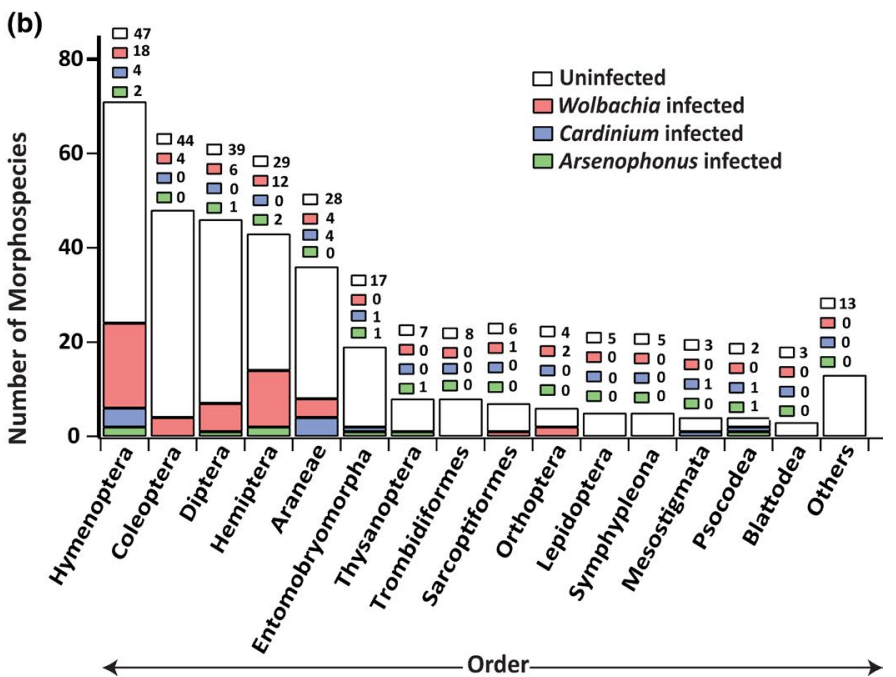

FIGURE 1 (a) Rarefaction curve of morphospecies found (in black) showing species richness in the soil arthropod community. Colored lines represent expected number of morphospecies. (b) The distribution of three endosymbionts screened across different host arthropod orders 


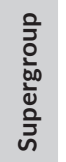

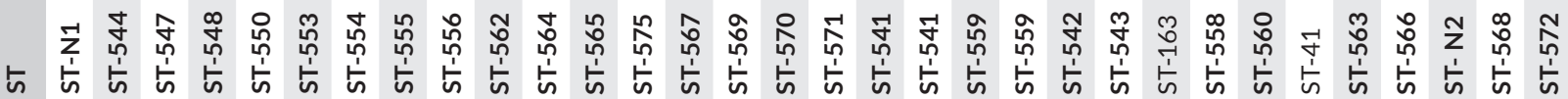

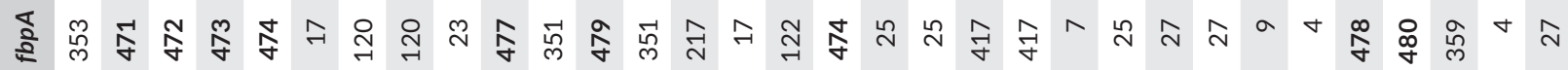

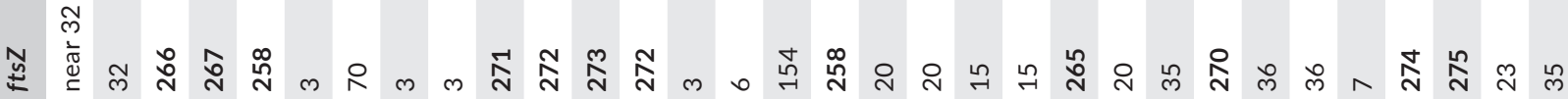
喜 今ิ 通

芯 崖



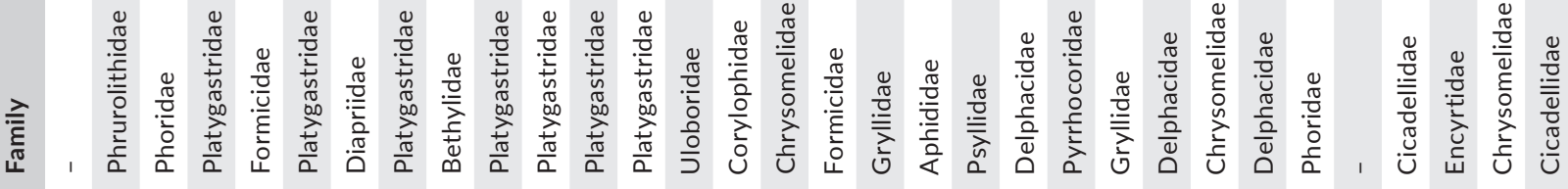

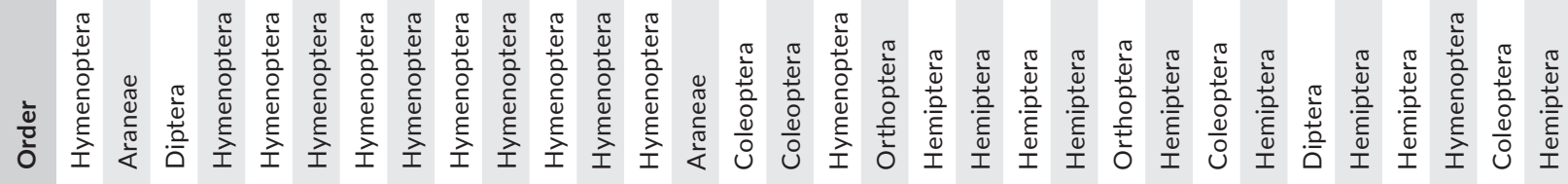
$\sum_{\frac{1}{\infty}}^{\stackrel{5}{c}}$

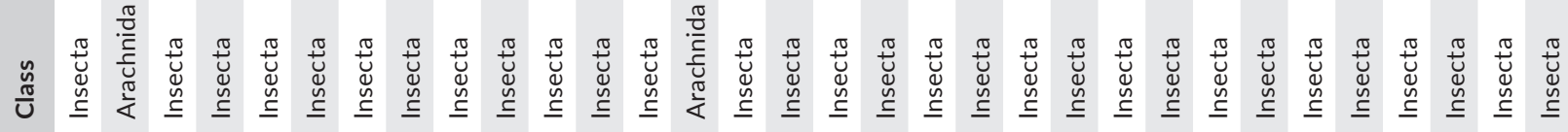
$\frac{\frac{2}{2}}{\frac{0}{\alpha}}$

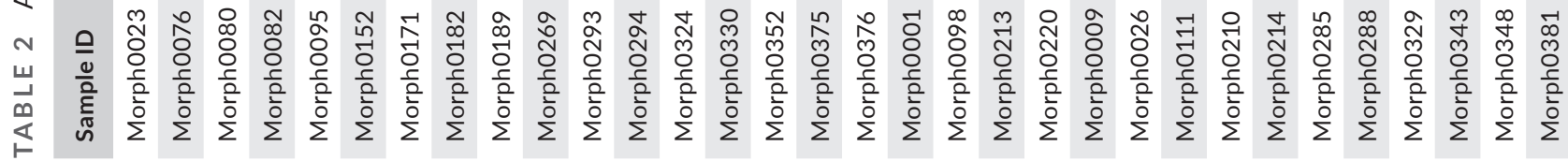




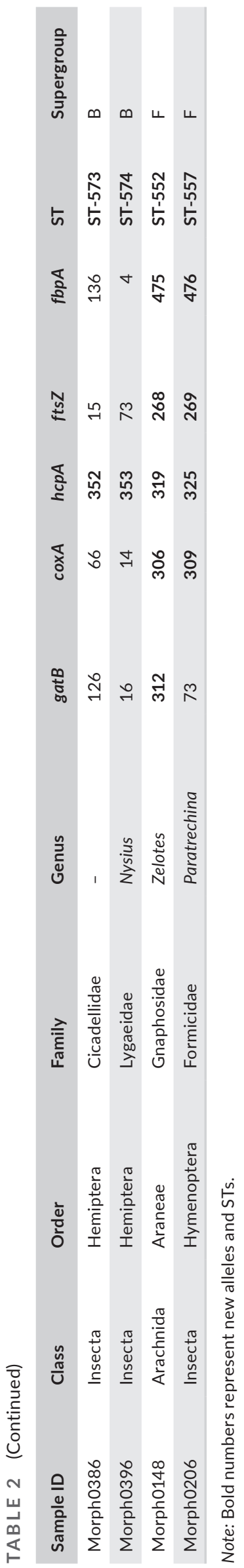

Similarly, another case of recombination was observed where a B supergroup Wolbachia ST-560, of morph0214 (HemipteraMuellerianella), had the coxA gene fragment (allele profile 2 ) from the A supergroup (Figure 4). This recombinant coxA allele 2 also shares sequence similarity with ST-550 and ST-571, where the coxA alleles are different by only two base pairs (coxA allele profile 305 ) indicating that perhaps this is also another case of recombination happening within the community.

Another case of recombination between supergroups was found with another MLST gene, gat $B$, but between supergroups $A$ and F. The Wolbachia ST-552 (supergroup F), infecting morph0148 (Araneae-Zelotes), had a recombinant gatB, where the last $190 \mathrm{bp}$ fragment came from the A supergroup. As the concatenated MLST tree (Figure 4) shows, ST-552 clusters with F supergroup, but the individual gatB gene tree shows it to be from the A supergroup. This 190 bp fragment differs by only one base pair with ST-544 infecting morph0076 (Araneae-Orthobula). This is also indicative of a possible recombination between these two Wolbachia STs belonging to two different supergroups.

\subsection{2 | Recombination within supergroups}

The pervasive recombination necessitated the development of the MLST scheme for Wolbachia (Baldo et al., 2006) as single-gene phylogenies were unable to properly represent the evolutionary history of a particular strain. In this scheme, alleles of any of the five different genes are given the same nomenclature if they share sequence identity. As Table 2 shows, many of the morphospecies also share the same alleles. In fact, instead of the maximum possible number of unique alleles (180) that could have been present across the five MLST loci of the 36 infected morphospecies, there is only 136. This is indicative of acquisition of the same alleles by recombination and is therefore examples of within-supergroup recombination events whereby MLST fragments are exchanged across endosymbionts.

Next, we tried to identify intergenic (i.e., within a particular MLST gene) recombination happening within a supergroup. This detection was done through the different algorithms present in RDP4, most of which uses inherently conservative sliding-window scans checking for above than expected sequence divergence in the alignments (Martin et al., 2015). Therefore, recombination events happening between closely related strains and/or between regions with low variation will not be recorded as significant events. However, there can be two types of intergenic recombination events. First, different MLST fragments (e.g., between coxA and gatB of two different strains) can combine to form a chimeric gene, and secondly, recombination can happen within the same MLST genes (e.g., within coxA of two different strains). Our analysis did not find any examples of the former. This is unsurprising as all the MLST fragments are housekeeping genes, and such chimeric variants will be under strong negative selection. However, eight instances of recombination within the same MLST gene were found (Table 3), all within supergroup A. 
Wolbachia

(a)

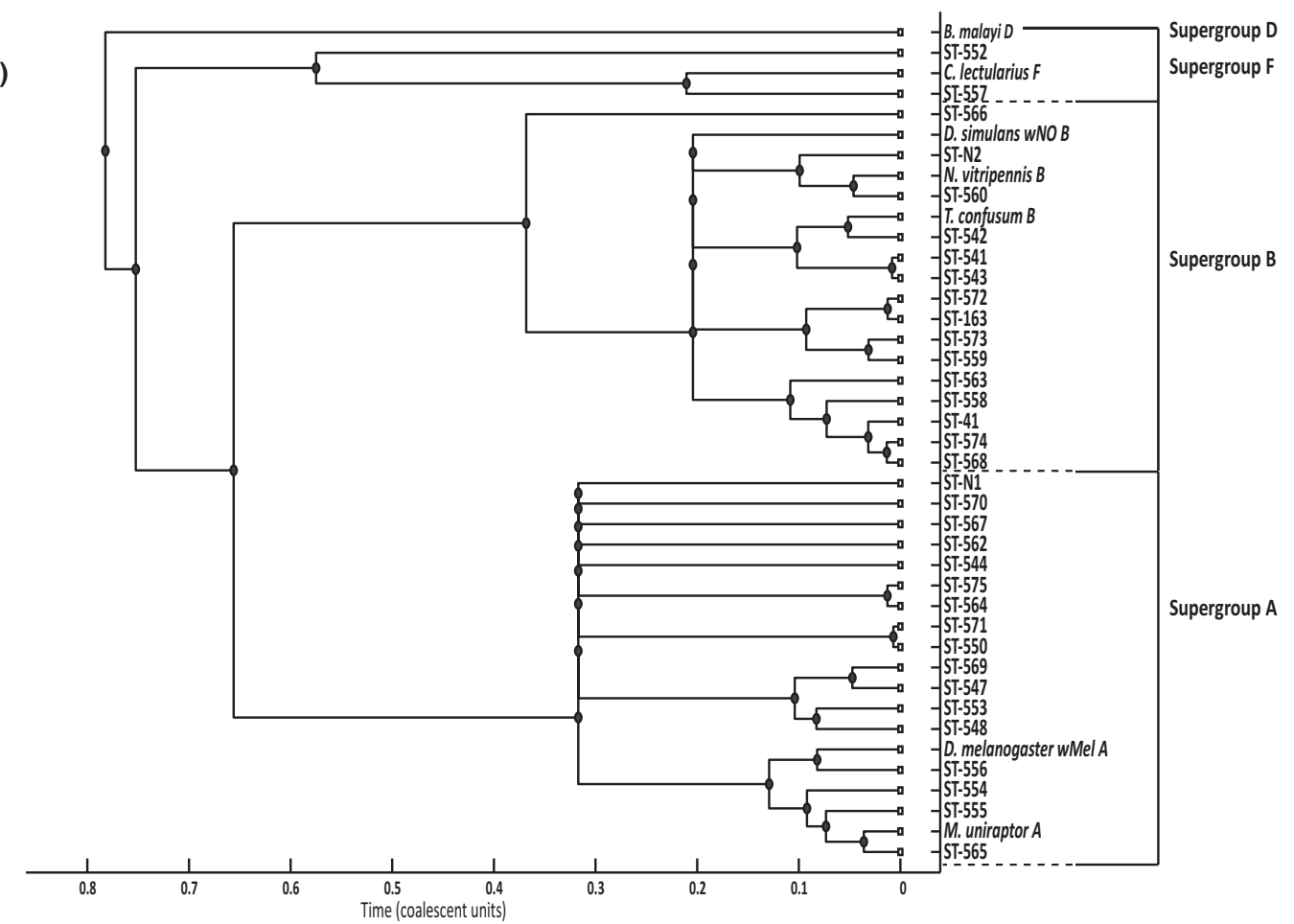

(b)

Cardinium

(c)

Arsenophonus
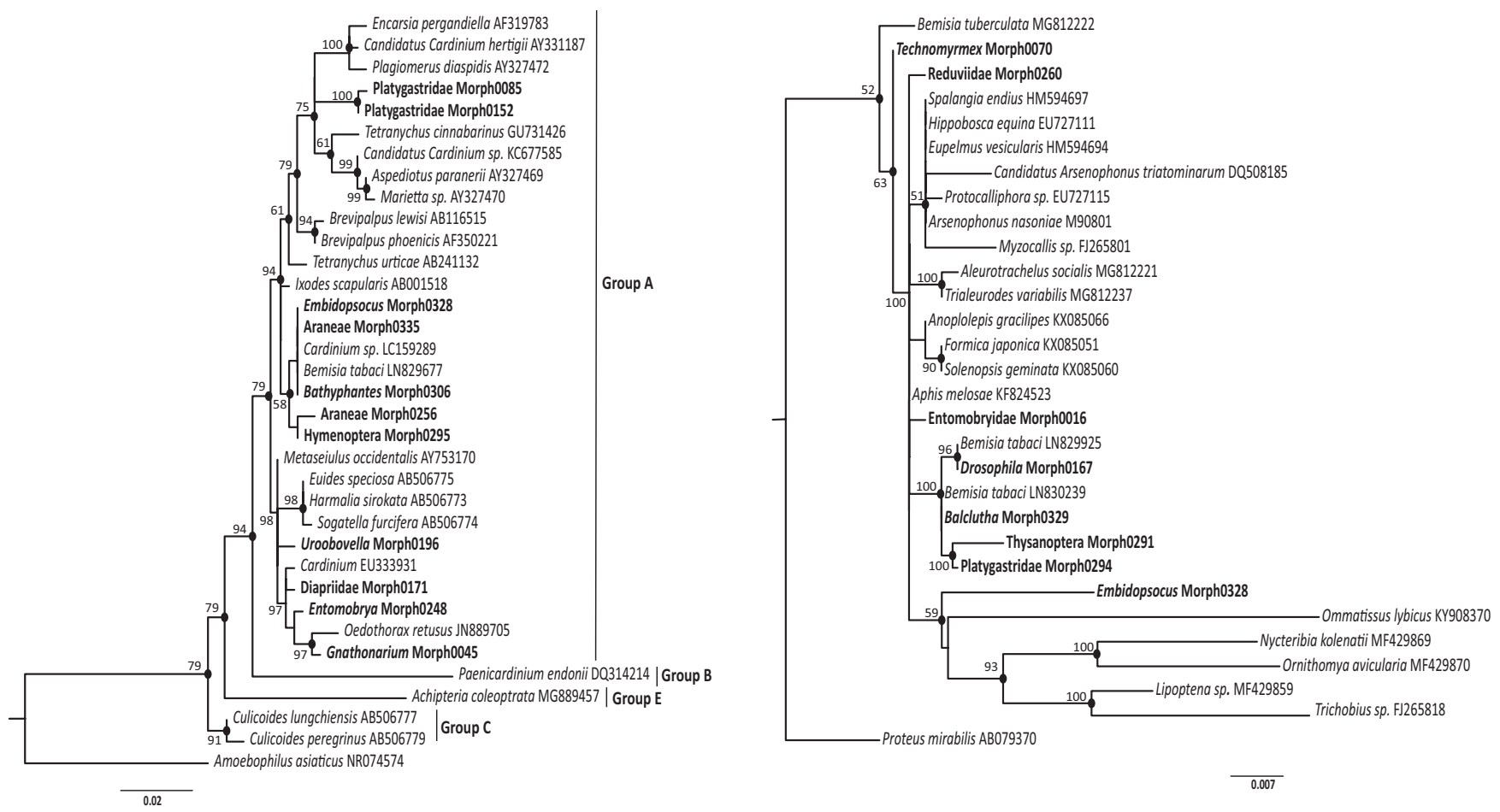

FIGURE 2 Phylogenetic analysis of (a) Wolbachia, (b) Cardinium, and (c) Arsenophonus found, shown with some known sequences for better resolution. Wolbachia phylogenetic tree was constructed using MLST data in ClonalFrame with at least $50 \%$ majority rule consensus. Cardinium and Arsenophonus phylogeny was made in MEGA7 using 16 S rRNA gene fragment using K2+g substitution model. Numbers on the nodes represent clade credibility values. Wolbachia infections are shown as STs whereas Cardinium and Arsenophonus are labeled with the host taxa that they infected. Infections obtained in this study are in bold. Brugia malayi, Amoebophilus asiaticus, and Proteus mirabilis were taken as out-group for Wolbachia, Cardinium, and Arsenophonus phylogenetic analyses, respectively 
(a)

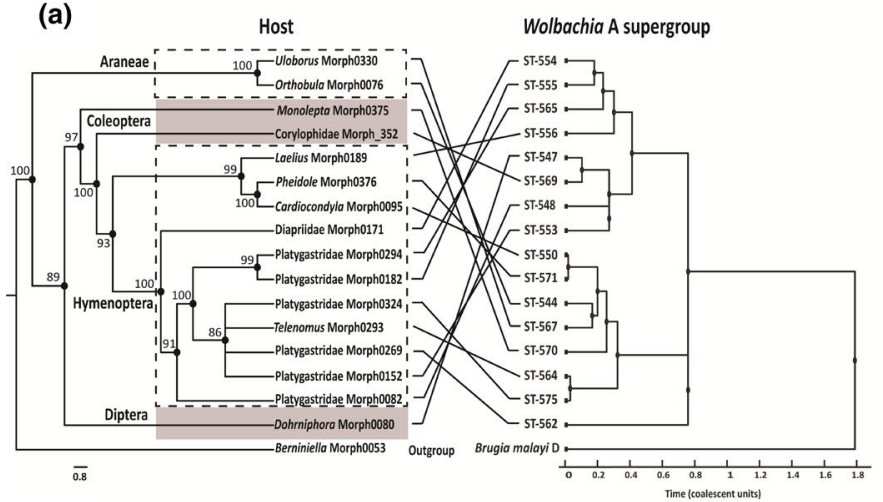

(c)

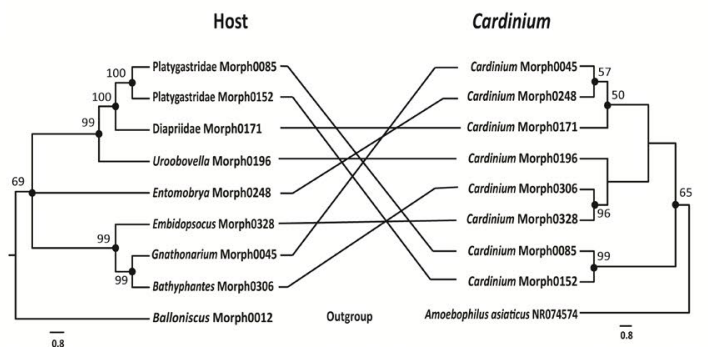

(b) Host Wolbachia B supergroup

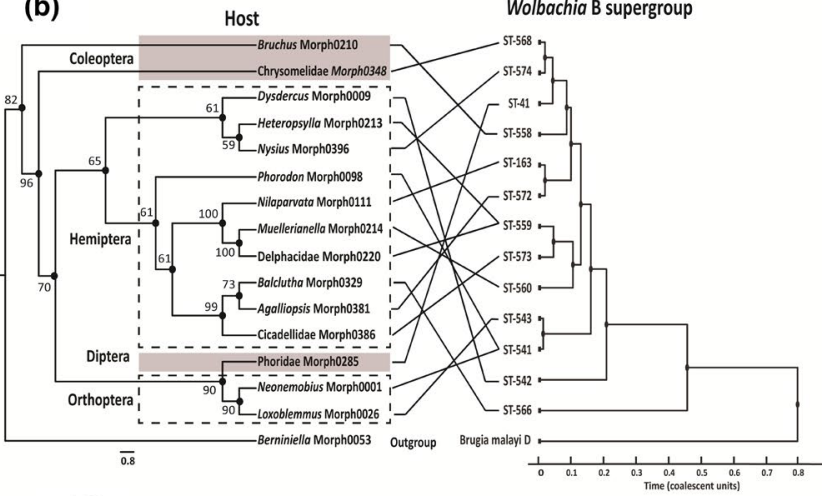

(d)

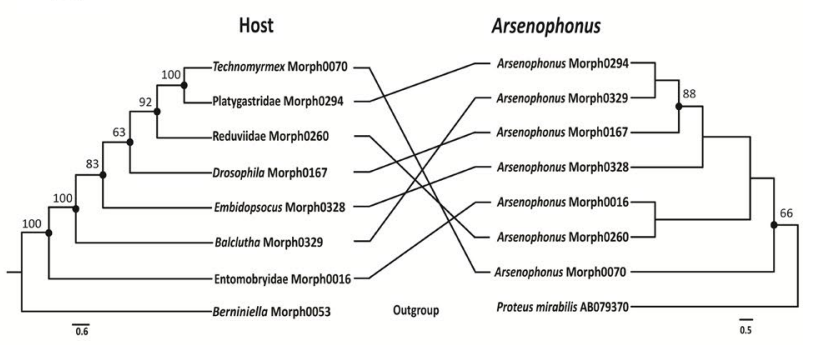

FIGURE 3 Association between infected host (left) and endosymbiont (right) phylogeny with (a) Wolbachia A supergroup, (b) Wolbachia B supergroup, (c) Cardinium, and (d) Arsenophonus. Host phylogeny was constructed in MrBayes using CO1 fragments. Phylogenetic relationship between different Wolbachia strains was interpreted through ClonalFrame with at least $50 \%$ majority rule consensus. Cardinium and Arsenophonus $16 \mathrm{~S} \mathrm{rDNA}$ phylogeny was constructed using MEGA7. Black dots in endosymbiont tree represent clade credibility $>50$

\subsubsection{How similar are endosymbiont strains within the community?}

If an ecological community is the primary site of horizontal transfer of endosymbionts, then the same (or very similar) bacterial strains would be found in multiple host taxa. This essentially means that a few endosymbiont strains within that community will show high incidence than other strains. This would result in a lower estimate of pairwise divergence among the endosymbionts. Using the model from Baldo et al. (2008), we tested whether there is a significant reduction in the expected pairwise divergence of the endosymbionts infecting the soil arthropod community. From our sampling, we found 17 unique STs for Wolbachia supergroup A and 15 for Wolbachia supergroup $B$ infections, whereas from the PubMLST database, we obtained 228 unique STs for A supergroup and 252 STs for B supergroup (last checked May 2019). Similarly, for the nine samples infected with Cardinium and eight for Arsenophonus, 248 and 228 sequences, respectively, were obtained from NCBI (last checked May, 2020). Results indicate that mean pairwise distance of Wolbachia supergroup A within the community (2.67\%) was significantly lower (Wilcox rank-sum test, $p<0.05$ ) than expected (mean $=3.54 \%$; Table S4) and also significantly lower from the mean of all the supergroup A strains present in the PubMLST database $3.69 \%$; Figure S7). In contrast, the mean pairwise distance of Wolbachia supergroup B strains within the community (4.17\%) was significantly higher (Wilcox rank-sum test, $p<0.05$ ) from both the expected (mean $=3.38 \%$ ) and the mean of all the B supergroup strains in the PubMLST database (3.43\%). These higher than expected values for
Wolbachia B supergroup strains can indicate the presence of more divergent strains as compared to Wolbachia A supergroup within this community. However, when all the Wolbachia supergroup infections were taken together and their mean pairwise distance (8.68\%) was compared with all such strains in the PubMLST database (8.66\%), no significant differences were found (Wilcox rank-sum test, $p>0.05$ ). This perhaps indicates that although the soil arthropod community yielded several unique Wolbachia infections (Table 2), on average, this still represents a subset of the Wolbachia diversity reported till now. Similar to Wolbachia supergroup A, Cardinium strains also showed a similar trend where community mean pairwise distance $(1.41 \%)$ was significantly less (Wilcox rank-sum test, $p<0.05$ ) than expected (mean $=2.48 \%$ ) and mean of strains obtained from the database (2.01\%). However, mean pairwise distance of Arsenophonus strains within the community (1.19\%) was not significantly different $(p>0.05)$ from the expected (mean $=1.38 \%$ ) as well as from mean of strains obtained from the database (1.55\%). Thus, Wolbachia supergroup $A$ and Cardinium strains within the community are more closely related among themselves (Table S4) but not Wolbachia supergroup B and Arsenophonus.

\section{4 | DISCUSSION}

In this study, we evaluated sequence divergence and incidence of recombination in three major endosymbionts (Wolbachia, Cardinium, and Arsenophonus) to answer whether the ecological community represents the primary seat of their horizontal transfer and 
$(\operatorname{cox} A)$



Concatenated MLST (gatB- coxA- hcpA-ftsZ- fbpA)

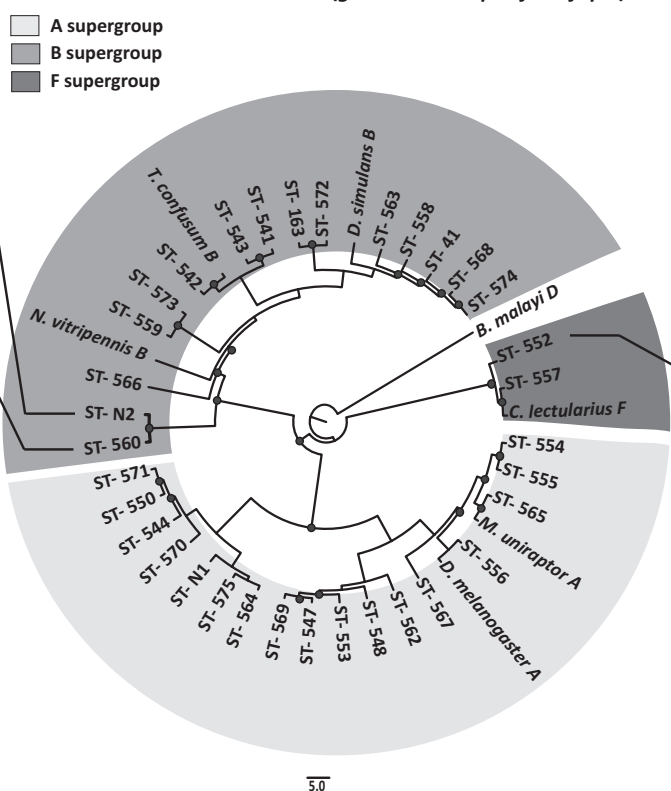

(gatB)



FIGURE 4 Maximum-likelihood phylogenetic trees of coxA (left), concatenated MLST dataset (center), and gatB (right) gene made in MEGA7 using HKY+g, T92+g+l, T92+g substitution models, respectively. Black dots represent bootstrap value $>50$. Wolbachia ST-N2 and ST-560 clustered with B supergroup in concatenated MLST phylogenetic tree whereas these strains clustered with A supergroup in the coxA phylogenetic tree, indicating recombination between Wolbachia supergroups A and B. Similarly, ST-552 clustered with F supergroup in concatenated MLST tree, but the individual gat $B$ gene tree shows it to be from the A supergroup, indicating recombination between $A$ and $F$ supergroups. These three cases (ST-N2, ST-560, and ST-552) represent between-supergroup recombination of gene or gene segment

diversification. We used soil arthropod community because it is relatively insular and has a relatively high habitat endemicity of the resident hosts. Our main goal was to assess whether community members facilitate the spread of endosymbionts as they themselves come in contact with each other for various ecological interactions. To do so, we compared multigene phylogenies of such endosymbiont surveys from different communities. However, in spite of our extensive literature surveys we could not find any such previous reports. Most surveys of arthropod communities concentrated on the hosts rather than on their endosymbionts (Gonçalves et al., 2012). Some studies such as Kittayapong et al. (2003) and Sintupachee et al. (2006) did uncover the resident endosymbionts but mostly with single genes. This precluded a cogent comparison of endosymbiont diversity and incidence of recombination with the present study. Another set of studies did indeed sample endosymbionts with multigene sequences but concentrated on a few, and not all, host taxa within a community (Bing et al., 2014). Again, such studies are not ideal comparisons with the present one as these were biased toward a few host taxa. To partially overcome this problem, we used statistical models with extensive resampling. We observed that the supergroup A Wolbachia infections and Cardinium do indeed show less pairwise divergence, than expected, in accordance with our predictions. However, supergroup B Wolbachia and Arsenophonus infections did not show this pattern. In fact, the former shows more variation than expected whereas Arsenophonus shows no significant difference. This indicates that these endosymbionts have different propensity and/or rates of horizontal transfer within the community than supergroup A Wolbachia and Cardinium. This disparity among the endosymbionts was still detectable in spite of our conservative estimate of the number endosymbionts within the community as we only analyzed hosts with single Wolbachia infections. This was primarily done to avoid the difficulty in assigning specific MLST alleles to individual bacterial strains.

\section{1 | Are some endosymbionts more prone to horizontal transfer and recombination?}

One explanation for the observed patterns could be the relative ease with which supergroup A Wolbachia and Cardinium can undergo horizontal transfer and recombination compared with supergroup $B$ Wolbachia and Arsenophonus. This essentially means that the former two endosymbionts would encounter previously existing bacterial infections within their hosts which would increase opportunities for recombination among the pre-existing and the new bacterial strains. Recombination would then create newer allele variants. This is indeed borne out by the results in Table 2 which depicts the number of unique alleles found in this study among the Wolbachia infections. In all, about $84 \%$ (71 out of a possible 85 alleles) of the A supergroup infection are unique, whereas about $75 \%$ (56 out of a possible 75 alleles) are unique in B supergroup Wolbachia infections. Furthermore, as indicated in Table 3, the number of within-supergroup recombination detected in the A supergroup strains (8 instances) far outnumbers the B supergroup Wolbachia, where none were detected. This is 
in spite of horizontal transfer of the entire B supergroup STs (ST-541 and ST-559) to taxonomically unrelated hosts (Table 2). An expected outcome of such pervasive horizontal transfer and resulting recombination would have been an increase in sequence diversity in the A supergroup strains, especially, if the source of recombination had been infections outside the community. This does not seem to be the case as the A supergroup infection shows less than expected pairwise distance $(2.67 \%)$ when compared with the B supergroup infections (4.17\%). This indicates that the sources of recombination must be from infections within this community. In other words, the standing sequence variation of the A supergroup infections is being partitioned across the community-wide arthropod taxa into newer recombinants with resulting increase in allele diversity but not overall sequence divergence. Moreover, what follows from this relatively low pairwise divergence of the A supergroup infections is that this horizontal transfer and recombination must have been relatively recent or rapid enough for any post-recombination sequence variation to accumulate. This indicates that the $A$ supergroup infections are either better at horizontal transfer across the community or are presently undergoing such rapid transfers as has been suggested by Werren et al. (1995). Whether this transfer is due to the presence of dominant strains, which are better at horizontal transfer (Turelli et al., 2018), remains to be investigated with more sequence information from these endosymbionts. The B supergroup Wolbachia infections show relatively diverged strains with low rates of recombination indicating more stable infections. Since little is known about the biological characteristics of Wolbachia supergroups, other than sequence divergence, it is difficult to speculate whether there are supergroup-specific effects on their hosts. For example, it is not known whether supergroup infections have different success rates in establishing stable infections after horizontal transfer or whether some are more prone to horizontal transfer? Therefore, we concentrated on specific trophic interactions of the hosts themselves and tried to explain why supergroup A infections show such extensive horizontal spread.

\subsection{Are parasitoids serving as the conduit for the spread of endosymbionts?}

Parasitoids can serve as a driving force for the horizontal transfer of endosymbionts (Haine et al., 2005) as their lifestyle entails close cellular and tissue contact with their host. Horizontal transfer involving parasitoids is generally unidirectional (from host to parasitoid) because they usually end up killing the host. But parasitoids can also act as phoretic vectors and can transmit endosymbionts by sequential probing of infected and uninfected hosts (Ahmed et al., 2015; Gehrer \& Vorburger, 2012). Moreover, horizontal transfers can also happen between parasitoids if infected and uninfected parasitoids share the same host (Huigens et al., 2004). Such habits can also facilitate multiple infections if parasitoids infecting same host have different endosymbiont infections. As these multiple infections come in close contact, they can undergo recombination. Such parasitoidmediated horizontal transfer could be an explanation for the distribution of $A$ supergroup Wolbachia infections in our sampling. Out of the 17 Wolbachia A supergroup STs found, nine STs were found in parasitoid wasps (Hymenoptera). Seven STs were found from Platygastridae and one each from Bethylidae and Diapriidae (Table 2). Therefore, the comparatively higher incidence of recombination in Wolbachia supergroup A infections could be due to their presence in parasitoid hosts. Similarly, predators and parasites can also be conduits for the spread of endosymbionts as observed during predation of infected Armadillidium vulgare by Porcellio dilatatus (Le Clec'h et al., 2013). Among the soil arthropod community, we also observed incidences of Wolbachia recombination among predators. Wolbachia F supergroup ST-552, infecting morph0148 (Araneae-Zelotes), had

TABLE 3 Recombination events detected in the Wolbachia MLST sequences. Putative breakpoints indicate concatenated sequences of MLST genes in the order: gatB-coxA-hcpA-ftsZ-fbpA. $p$-value was kept at $<.01$

\begin{tabular}{|c|c|c|c|c|}
\hline Recombination event & & $\begin{array}{l}\text { STs potentially } \\
\text { involved }\end{array}$ & $\begin{array}{l}\text { Gene: Putative } \\
\text { breakpoints }\end{array}$ & $\begin{array}{l}\text { Detection methods positive } \\
\text { for recombination }\end{array}$ \\
\hline \multirow[t]{3}{*}{ Between supergroup } & $A$ and $B$ supergroups & ST-N2/565 & $\operatorname{cox} A: 370-771$ & $\begin{array}{l}\text { RDP, GENECONV, BootScan, } \\
\text { MaxChi, Chimera, SiScan }\end{array}$ \\
\hline & $A$ and $B$ supergroups & ST-560/550 & coxA: 370-771 & $\begin{array}{l}\text { RDP, GENECONV, BootScan, } \\
\text { MaxChi, Chimera }\end{array}$ \\
\hline & $A$ and $F$ supergroups & ST-544/552 & gatB: 179-369 & $\begin{array}{l}\text { RDP, GENECONV, BootScan, } \\
\text { 3Seq }\end{array}$ \\
\hline \multirow[t]{5}{*}{ Within supergroup } & A supergroup & ST-565/555 & gatB: $255-369$ & MaxChi, SiScan, 3 Seq \\
\hline & & & fbpA: $1650-1800$ & \\
\hline & A supergroup & ST-547/564 & gatB: $118-369$ & MaxChi, Chimera, SiScan, 3 Sea \\
\hline & & & hсрA: 771-885 & \\
\hline & A supergroup & ST-N1/567 & gatB: 80-369 & Chimera, SiScan, 3Sea \\
\hline
\end{tabular}


a fragment of gatB gene similar to the A supergroup from ST-544 which infected morph0076 (Araneae-Orthobula). Furthermore, the fbpA gene of ST-544 was found to have probably recombined with ST-570 (Morph0375, Coleoptera-Monolepta sp.). Compared to predation, host-parasitoid interactions generally last longer can take place at various developmental stages and allow endosymbiotic bacteria to enter host through various tissues (Sanaei et al., 2021). These features can increase the probability of parasitoid-mediated horizontal transfer which remains one of the most common routes for endosymbiont spread (Hou et al., 2020; Morrow et al., 2014). Therefore, occurrence of closely related A supergroup Wolbachia among the parasitoid hosts of this community is not unexpected. However, this pattern can also be explained by Wolbachia A supergroup infections being "better" at horizontal transfer and subsequent establishment in a new host. Further studies are required to answer which of these two explanations are more plausible.

\subsection{How are endosymbionts spreading from one ecological community to another?}

Ecological communities are a diverse assemblage of many different species involved in a web of interactions with each other (Agrawal et al., 2007). However, rarely, such communities remain isolated from each other. There are certain members which are relatively cosmopolitan and interconnect with members of multiple communities (Stireman \& Singer, 2003) leading to a metacommunity-wide distribution (Brown et al., 2020). This cosmopolitan distribution of these arthropods can lead to horizontal transfer of their resident endosymbionts to different ecological communities. Within the soil arthropod community, we have found one such example which can potentially be a source of horizontal transfer of endosymbionts across many other communities. The macropterous form of the planthopper Nilaparvata lugens (morph0111, BOLD ID SAEVG089-20; Table S2) was found from the leaf litter. N. lugens is a highly destructive pest of rice across tropical Asia and can also survive on other tropical grass species (Khan et al., 1988). It is known to migrate long distances in search of actively growing rice plants (Riley et al., 2003). The presence of $N$. lugens is unsurprising as our sampling season (October) coincided with the rice harvesting season in Northwest India. N. lugens is known to be infected with several endosymbionts such as Wolbachia and Arsenophnonus (Qu et al., 2013). In this study, it was found to be infected with Wolbachia ST-163 from the B supergroup. The same Wolbachia ST has also been reported in N. lugens from Southern China (Zhang et al., 2013). This indicates that such invasive pest species can potentially introduce their resident endosymbionts into many different arthropod communities.

Conversely, the presence of very similar endosymbionts in geographically distinct locations can indicate their spread from one ecological community to another. The Wolbachia B supergroup, ST41, was found to infect a phorid fly (morph0285). The same ST-41 has been found in calyptrate flies (Stahlhut et al., 2010) as well as from several other lepidopterans (Ilinsky \& Kosterin, 2017; Narita et al., 2011; Russell et al., 2009; Salunke et al., 2012). This is not unexpected given the diversity of Wolbachia infections. However, what is unexpected is the location of the hosts with ST-41 ranges from North America, Africa, Russia, South Asia, and South-Eastern Asia all the way to Japan. Unfortunately, it is difficult to conjecture about the reasons behind such a huge range, as corroborating communitywide data are lacking.

The above two instances testify to the utility of a MLST-based approach to understand Wolbachia diversity and spread across global arthropod communities (Wang et al., 2020). Moreover, these cases also highlight the importance of collecting community-wide data to understand the probable chain of transfer of these bacteria. Such data can also illuminate similar connections for the spread of Arsenophonus and Cardinium if employed with multilocus data (Jousselin et al., 2013; Stouthamer et al., 2019).

A major goal of endosymbiont research is to explain the tempo and mode of their spread across arthropod communities across the world. We contend that evaluating endosymbiont diversity within specific ecological communities is the key to understand this spread. Such studies would give us specific examples of bacterial strains that are better at spreading as well as uncover specific ecological roles of arthropod hosts which are more amenable to horizontal transfer of their resident endosymbionts. As data from such studies accumulate specific patterns will emerge which can then be empirically tested.

\section{ACKNOWLEDGEMENTS}

We would like to thank IISER-Mohali for providing the experimental facilities and funding for this work. We acknowledge all EVOGEN lab members for their comments on manuscript. MG thank University Grant Commission (UGC) for fellowship (ID: 325974). AG is grateful to ICAR for necessary facilities. RK was funded by postdoctoral fellowship from IISER-Mohali. We would like to thank Professor Raman Rajagopal, Department to Zoology, University of Delhi, for providing us with Cardinium and Arsenophonus positive controls and Yogesh Bangal for help in field sampling.

\section{CONFLICT OF INTEREST}

None declared.

\section{AUTHOR CONTRIBUTIONS}

Manisha Gupta: Conceptualization (equal); data curation (lead); formal analysis (lead); investigation (lead); methodology (lead); software (lead); validation (lead); visualization (equal); writing-original draft (equal); writing-review and editing (equal). Rajbir Kaur: Investigation (equal); methodology (equal); visualization (equal). Ankita Gupta: Data curation (supporting); investigation (supporting). Rhitoban Raychoudhury: Conceptualization (lead); formal analysis (equal); funding acquisition (lead); investigation (lead); methodology (lead); project administration (lead); supervision (lead); validation (equal); visualization (equal); writing-original draft (equal); writing-review and editing (equal). 


\section{DATA AVAILABILITY STATEMENT}

All sequences obtained from this study were deposited in NCBI. Accession numbers are as follows: for CO1 data-MN447522MN447531, MN520845-MN521147, MN901899; Wolbachia gatB-MN594583-MN594618; $\quad$ coxA-MN594619-MN594654; hcpA-MN594655-MN594690; ftsZ-MN594691-MN594726; fbpAMN594727-MN594762; Cardinium 16S-MN594564-MN594574; and Arsenophonus 16S-MN594575-MN594582 (Table S2). Wolbachia MLST data were also deposited on PubMLST database having ID ST541-544, 547-548, 550, 552-560, 562-575 (Table 2). Morphospecies images along with their corresponding $\mathrm{CO} 1$ gene sequences were also deposited in BOLD database having process ID SAEVG001-20: SAEVG314-20.

\section{ORCID}

Rhitoban Raychoudhury (iD https://orcid. org/0000-0001-6835-1008

\section{REFERENCES}

Agrawal, A. A., Ackerly, D. D., Adler, F., Arnold, A. E., Cáceres, C., Doak, D. F., Post, E., Hudson, P. J., Maron, J., Mooney, K. A., Power, M., Schemske, D., Stachowicz, J., Strauss, S., Turner, M. G., \& Werner, E. (2007). Filling key gaps in population and community ecology. Frontiers in Ecology and the Environment, 5(3), 145-152. https://doi. org/10.1890/1540-9295(2007)5[145:FKGIPA]2.0.CO;2

Ahmed, M. Z., Breinholt, J. W., \& Kawahara, A. Y. (2016). Evidence for common horizontal transmission of Wolbachia among butterflies and moths. BMC Evolutionary Biology, 16(1), 1-16. https://doi. org/10.1186/s12862-016-0660-x

Ahmed, M. Z., Li, S.-J., Xue, X., Yin, X.-J., Ren, S.-X., Jiggins, F. M., Greeff, J. M., \& Qiu, B.-L. (2015). The intracellular bacterium Wolbachia uses parasitoid wasps as phoretic vectors for efficient horizontal transmission. PLoS Pathogen, 11(2), e1004672. https://doi.org/10.1371/ journal.ppat.1004672

Amancio, G., Aguirre-Jaimes, A., Hernández-Ortiz, V., Guevara, R., \& Quesada, M. (2019). Vertical and horizontal trophic networks in the aroid-infesting insect community of Los Tuxtlas biosphere reserve, Mexico. Insects, 10(8), 252. https://doi.org/10.3390/insects100 80252

Bailly-Bechet, M., Martins-Simões, P., Szöllősi, G. J., Mialdea, G., Sagot, M.-F., \& Charlat, S. (2017). How long does Wolbachia remain on board? Molecular Biology and Evolution, 34(5), 1183-1193. https:// doi.org/10.1093/molbev/msx073

Balbuena, J. A., Míguez-Lozano, R., \& Blasco-Costa, I. (2013). PACo: A novel procrustes application to cophylogenetic analysis. PLoS One, 8(4), e61048. https://doi.org/10.1371/journal.pone.0061048

Baldo, L., Ayoub, N. A., Hayashi, C. Y., Russell, J. A., Stahlhut, J. K., \& Werren, J. H. (2008). Insight into the routes of Wolbachia invasion: High levels of horizontal transfer in the spider genus Agelenopsis revealed by Wolbachia strain and mitochondrial DNA diversity. Molecular Ecology, 17(2), 557-569. https://doi. org/10.1111/j.1365-294X.2007.03608.x

Baldo, L., Bordenstein, S., Wernegreen, J. J., \& Werren, J. H. (2006). Widespread recombination throughout Wolbachia genomes. Molecular Biology and Evolution, 23(2), 437-449. https://doi. org $/ 10.1093 / \mathrm{molbev} / \mathrm{msj049}$

Baldo, L., Dunning Hotopp, J. C., Jolley, K. A., Bordenstein, S. R., Biber, S. A., Choudhury, R. R., \& Werren, J. H. (2006). Multilocus sequence typing system for the endosymbiont Wolbachia pipientis. Applied and Environment Microbiology, 72(11), 7098-7110.
Baudry, E., Bartos, J., Emerson, K., Whitworth, T., \& Werren, J. H. (2003). Wolbachia and genetic variability in the birdnest blowfly Protocalliphora sialia. Molecular Ecology, 12(7), 1843-1854.

Bing, X. L., Xia, W. Q., Gui, J. D., Yan, G. H., Wang, X. W., \& Liu, S. S. (2014). Diversity and evolution of the Wolbachia endosymbionts of Bemisia (Hemiptera: Aleyrodidae) whiteflies. Ecology and Evolution, 4(13), 2714-2737.

Boni, M. F., Posada, D., \& Feldman, M. W. (2007). An exact nonparametric method for inferring mosaic structure in sequence triplets. Genetics, 176(2), 1035-1047. https://doi.org/10.1534/genet ics.106.068874

Brown, A. N., \& Lloyd, V. K. (2015). Evidence for horizontal transfer of Wolbachia by a Drosophila mite. Experimental and Applied Acarology, 66(3), 301-311. https://doi.org/10.1007/s10493-015-9918-z

Brown, J. J., Mihaljevic, J. R., Des Marteaux, L., \& Hrček, J. (2020). Metacommunity theory for transmission of heritable symbionts within insect communities. Ecology and Evolution, 10(3), 1703-1721. https://doi.org/10.1002/ece3.5754

Bruen, T. C., Philippe, H., \& Bryant, D. (2006). A simple and robust statistical test for detecting the presence of recombination. Genetics, 172(4), 2665-2681. https://doi.org/10.1534/genet ics.105.048975

Bryant, D., \& Moulton, V. (2004). Neighbor-net: An agglomerative method for the construction of phylogenetic networks. Molecular Biology and Evolution, 21(2), 255-265. https://doi.org/10.1093/ molbev/msh018

Chao, A. (1984). Nonparametric estimation of the number of classes in a population. Scandinavian Journal of Statistics, 11, 265-270.

Chao, A., Hwang, W.-H., Chen, Y., \& Kuo, C. (2000). Estimating the number of shared species in two communities. Statistica Sinica, 10, 227-246.

Colwell, R. K. (2013). EstimateS: Statistical estimation of species richness and shared species from samples. Version 9 and earlier. User's Guide and application. Retrieved from http://purl.oclc.org/estimates

Dewayne Shoemaker, D., Machado, C. A., Molbo, D., Werren, J. H., Windsor, D. M., \& Herre, E. A. (2002). The distribution of Wolbachia in fig wasps: Correlations with host phylogeny, ecology and population structure. Proceedings of the Royal Society of London. Series B: Biological Sciences, 269(1506), 2257-2267.

Didelot, X., \& Falush, D. (2007). Inference of bacterial microevolution using multilocus sequence data. Genetics, 175(3), 1251-1266. https://doi.org/10.1534/genetics.106.063305

Duplouy, A., \& Hornett, E. A. (2018). Uncovering the hidden players in Lepidoptera biology: The heritable microbial endosymbionts. PeerJ, 6, e4629. https://doi.org/10.7717/peerj.4629

Duplouy, A., Vermenot, C., Davies, N., Roderick, G., Hurst, G. D., \& Charlat, S. (2009). Assessing risks of Wolbachia DNA crossspecimen contamination following mass collection and ethanol storage. Molecular Ecology Resources, 9(1), 46-50.

Duron, O., Bouchon, D., Boutin, S., Bellamy, L., Zhou, L., Engelstädter, J., \& Hurst, G. D. (2008). The diversity of reproductive parasites among arthropods: Wolbachia do not walk alone. BMC Biology, 6(1), 27. https://doi.org/10.1186/1741-7007-6-27

Ellegaard, K. M., Klasson, L., Naslund, K., Bourtzis, K., \& Andersson, S. G. (2013). Comparative genomics of Wolbachia and the bacterial species concept. PLoS Genetics, 9(4), e1003381. https://doi. org/10.1371/journal.pgen.1003381

Gebiola, M., Giorgini, M., Kelly, S. E., Doremus, M. R., Ferree, P. M., \& Hunter, M. S. (2017). Cytological analysis of cytoplasmic incompatibility induced by Cardinium suggests convergent evolution with its distant cousin Wolbachia. Proceedings of the Royal Society B: Biological Sciences, 284(1862), 20171433.

Gehrer, L., \& Vorburger, C. (2012). Parasitoids as vectors of facultative bacterial endosymbionts in aphids. Biology Letters, 8(4), 613-615. https://doi.org/10.1098/rsbl.2012.0144 
Gerth, M., Röthe, J., \& Bleidorn, C. (2013). Tracing horizontal Wolbachia movements among bees (Anthophila): A combined approach using multilocus sequence typing data and host phylogeny. Molecular Ecology, 22(24), 6149-6162.

Gibbs, M. J., Armstrong, J. S., \& Gibbs, A. J. (2000). Sister-scanning: A Monte Carlo procedure for assessing signals in recombinant sequences. Bioinformatics, 16(7), 573-582. https://doi.org/10.1093/ bioinformatics/16.7.573

Gonçalves, M. F., Pereira, J. A., \& Liu, T. (2012). Abundance and diversity of soil arthropods in the olive grove ecosystem. Journal of Insect Science, 12(1), 20. https://doi.org/10.1673/031.012.2001

Gonella, E., Pajoro, M., Marzorati, M., Crotti, E., Mandrioli, M., Pontini, M., Bulgari, D., Negri, I., Sacchi, L., Chouaia, B., Daffonchio, D., \& Alma, A. (2015). Plant-mediated interspecific horizontal transmission of an intracellular symbiont in insects. Scientific Reports, 5, 110. https://doi.org/10.1038/srep15811

Gotoh, T., Noda, H., \& Ito, S. (2007). Cardinium symbionts cause cytoplasmic incompatibility in spider mites. Heredity, 98(1), 13-20. https:// doi.org/10.1038/sj.hdy.6800881

Haine, E. R., Pickup, N. J., \& Cook, J. M. (2005). Horizontal transmission of Wolbachia in a Drosophila community. Ecological Entomology, 30(4), 464-472. https://doi.org/10.1111/j.0307-6946.2005.00715.x

Hall, T. A. (1999). BioEdit: A user-friendly biological sequence alignment editor and analysis program for Windows 95/98/NT. Nucleic Acids Symposium Series, 41, 95-98.

Hebert, P. D., Cywinska, A., Ball, S. L., \& Dewaard, J. R. (2003). Biological identifications through DNA barcodes. Proceedings of the Royal Society of London. Series B: Biological Sciences, 270(1512), 313-321. https://doi.org/10.1098/rspb.2002.2218

Hilgenboecker, K., Hammerstein, P., Schlattmann, P., Telschow, A., \& Werren, J. H. (2008). How many species are infected with Wolbachia?-A statistical analysis of current data. FEMS Microbiology Letters, 281(2), 215-220.

Hou, H. Q., Zhao, G. Z., Su, C. Y., \& Zhu, D. H. (2020). Wolbachia prevalence patterns: Horizontal transmission, recombination, and multiple infections in chestnut gall wasp-parasitoid communities. Entomologia Experimentalis Et Applicata, 168(10), 752-765.

Hoy, M. A., \& Jeyaprakash, A. (2005). Microbial diversity in the predatory mite Metaseiulus occidentalis (Acari: Phytoseiidae) and its prey, Tetranychus urticae (Acari: Tetranychidae). Biological Control, 32(3), 427-441. https://doi.org/10.1016/j.biocontrol.2004.12.012

Huigens, M., De Almeida, R., Boons, P., Luck, R., \& Stouthamer, R. (2004). Natural interspecific and intraspecific horizontal transfer of parthenogenesis-inducing Wolbachia in Trichogramma wasps. Proceedings of the Royal Society of London. Series B: Biological Sciences, 271(1538), 509-515.

Huson, D. H., Kloepper, T., \& Bryant, D. (2008). SplitsTree4.0-Computation of phylogenetic trees and networks. Bioinformatics, 14, 68-73.

Ilinsky, Y., \& Kosterin, O. E. (2017). Molecular diversity of Wolbachia in Lepidoptera: Prevalent allelic content and high recombination of MLST genes. Molecular Phylogenetics and Evolution, 109, 164-179. https://doi.org/10.1016/j.ympev.2016.12.034

Jiggins, F. M., von Der Schulenburg, J. H., Hurst, G. D., \& Majerus, M. E. (2001). Recombination confounds interpretations of Wolbachia evolution. Proceedings of the Royal Society B: Biological Sciences, 268(1474), 1423-1427.

Johnson, M., Zaretskaya, I., Raytselis, Y., Merezhuk, Y., McGinnis, S., \& Madden, T. L. (2008). NCBI BLAST: A better web interface. Nucleic Acids Research, 36, W5-9. https://doi.org/10.1093/nar/gkn201

Jolley, K. A., Bray, J. E., \& Maiden, M. C. (2018). Open-access bacterial population genomics: BIGSdb software, the PubMLST. org website and their applications. Wellcome Open Research, 3, 124.

Jousselin, E., Cœur d'Acier, A., Vanlerberghe-Masutti, F., \& Duron, O. (2013). Evolution and diversity of Arsenophonus endosymbionts in aphids. Molecular Ecology, 22(1), 260-270.
Khan, Z., Saxena, R., \& Rueda, B. (1988). Responses of rice-infesting and grass-infesting populations of Nilaparvata lugens (Homoptera: Delphacidae) to rice plants and Leersia grass and to their steam distillate extracts. Journal of Economic Entomology, 81(4), 1080-1088. https://doi.org/10.1093/jee/81.4.1080

Kittayapong, P., Jamnongluk, W., Thipaksorn, A., Milne, J., \& Sindhusake, C. (2003). Wolbachia infection complexity among insects in the tropical rice-field community. Molecular Ecology, 12(4), 1049-1060. https://doi.org/10.1046/j.1365-294X.2003.01793.x

Kumar, S., Stecher, G., \& Tamura, K. (2016). MEGA7: Molecular evolutionary genetics analysis version 7.0 for bigger datasets. Molecular Biology and Evolution, 33(7), 1870-1874. https://doi.org/10.1093/ molbev/msw054

Landmann, F. (2019). The Wolbachia Endosymbionts. Microbiol Spectr, 7(2), 139-153.

Le Clec'h, W., Chevalier, F. D., Genty, L., Bertaux, J., Bouchon, D., \& Sicard, M. (2013). Cannibalism and predation as paths for horizontal passage of Wolbachia between terrestrial isopods. PLoS One, 8(4), e60232. https://doi.org/10.1371/journal.pone.0060232

Legendre, P., Desdevises, Y., \& Bazin, E. (2002). A statistical test for hostparasite coevolution. Systematic Biology, 51(2), 217-234. https:// doi.org/10.1080/10635150252899734

Legendre, P., \& Legendre, L. F. (2012). Numerical ecology (Vol. 24). Elsevier.

Li, S. J., Ahmed, M. Z., Lv, N., Shi, P. Q., Wang, X. M., Huang, J. L., \& Qiu, B. L. (2017). Plant mediated horizontal transmission of Wolbachia between whiteflies. The ISME Journal, 11(4), 1019-1028. https:// doi.org/10.1038/ismej.2016.164

Librado, P., \& Rozas, J. (2009). DnaSP v5: A software for comprehensive analysis of DNA polymorphism data. Bioinformatics, 25(11), 14511452. https://doi.org/10.1093/bioinformatics/btp187

Maiden, M. C. J., Bygraves, J. A., Feil, E., Morelli, G., Russell, J. E., Urwin R., Zhang, Q., Zhou, J., Zurth, K., Caugant, D. A., Feavers, I. M., Achtman, M., \& Spratt, B. G. (1998). Multilocus sequence typing: A portable approach to the identification of clones within populations of pathogenic microorganisms. Proceedings of the National Academy of Sciences, 95(6), 3140-3145. https://doi.org/10.1073/ pnas.95.6.3140

Malloch, G., \& Fenton, B. (2005). Super-infections of Wolbachia in byturid beetles and evidence for genetic transfer between $A$ and $B$ supergroups of Wolbachia. Molecular Ecology, 14(2), 627-637. https://doi. org/10.1111/j.1365-294X.2005.02432.x

Martin, D. P., Murrell, B., Golden, M., Khoosal, A., \& Muhire, B. (2015). RDP4: Detection and analysis of recombination patterns in virus genomes. Virus Evolution, 1(1), vev003. https://doi.org/10.1093/ve/ vev003

Martin, D., Posada, D., Crandall, K., \& Williamson, C. (2005). A modified bootscan algorithm for automated identification of recombinant sequences and recombination breakpoints. AIDS Research and Human Retroviruses, 21(1), 98-102. https://doi.org/10.1089/ aid.2005.21.98

Martin, D., \& Rybicki, E. (2000). RDP: Detection of recombination amongst aligned sequences. Bioinformatics, 16(6), 562-563. https:// doi.org/10.1093/bioinformatics/16.6.562

Mascarenhas, R. O., Prezotto, L. F., Perondini, A. L., Marino, C. L., \& Selivon, D. (2016). Wolbachia in guilds of Anastrepha fruit flies (Tephritidae) and parasitoid wasps (Braconidae). Genet Mol Biol, 39(4), 600-610. https://doi.org/10.1590/1678-4685-gmb-2016-0075

Mohammed, M. A., Aman-Zuki, A., Yusof, S., Md-Zain, B. M., \& Yaakop, S. (2017). Prevalence and evolutionary history of endosymbiont Wolbachia (Rickettsiales: Anaplasmataceae) in parasitoids (Hymenoptera: Braconidae) associated with Bactrocera fruit flies (Diptera: Tephritidae) infesting carambola. Entomological Science, 20(1), 382-395.

Morrow, J. L., Frommer, M., Shearman, D., \& Riegler, M. (2014). Tropical tephritid fruit fly community with high incidence of shared 
Wolbachia strains as platform for horizontal transmission of endosymbionts. Environmental Microbiology, 16(12), 3622-3637.

Mouton, L., Thierry, M., Henri, H., Baudin, R., Gnankine, O., Reynaud, B., Zchori-Fein, E., Becker, N., Fleury, F., \& Delatte, H. (2012). Evidence of diversity and recombination in Arsenophonus symbionts of the Bemisia tabaci species complex. BMC Microbiology, 12(1), 1-15. https://doi.org/10.1186/1471-2180-12-S1-S10

Nakamura, Y., Kawai, S., Yukuhiro, F., Ito, S., Gotoh, T., Kisimoto, R., \& Noda, H. (2009). Prevalence of Cardinium bacteria in planthoppers and spider mites and taxonomic revision of "Candidatus Cardinium hertigii" based on detection of a new Cardinium group from biting midges. Applied and Environmental Microbiology, 75(21), 6757-6763.

Narita, S., Kageyama, D., Hiroki, M., Sanpei, T., Hashimoto, S., Kamitoh, T., \& Kato, Y. (2011). Wolbachia-induced feminisation newly found in Eurema hecabe, a sibling species of Eurema mandarina (Lepidoptera: Pieridae). Ecological Entomology, 36(3), 309-317. https://doi. org/10.1111/j.1365-2311.2011.01274.x

Olson, D. M. (1991). A comparison of the efficacy of litter sifting and pitfall traps for sampling leaf litter ants (Hymenoptera, Formicidae) in a tropical wet forest, Costa Rica. Biotropica, 23, 166-172. https:// doi.org/10.2307/2388302

Padidam, M., Sawyer, S., \& Fauquet, C. M. (1999). Possible emergence of new geminiviruses by frequent recombination. Virology, 265(2), 218-225. https://doi.org/10.1006/viro.1999.0056

Posada, D., \& Crandall, K. A. (2001). Evaluation of methods for detecting recombination from DNA sequences: Computer simulations. Proceedings of the National Academy of Sciences, 98(24), 1375713762. https://doi.org/10.1073/pnas.241370698

Qu, L.-Y., Lou, Y.-H., Fan, H.-W., Ye, Y.-X., Huang, H.-J., Hu, M.-Q., Zhu, Y.-N., \& Zhang, C.-X. (2013). Two endosymbiotic bacteria, Wolbachia and Arsenophonus, in the brown planthopper Nilaparvata lugens. Symbiosis, 61(1), 47-53. https://doi.org/10.1007/s1319 9-013-0256-9

Querner, P., \& Bruckner, A. (2010). Combining pitfall traps and soil samples to collect Collembola for site scale biodiversity assessments. Applied Soil Ecology, 45(3), 293-297. https://doi.org/10.1016/j. apsoil.2010.05.005

Rambaut, A. (2009). FigTree. Tree figure drawing tool. Retrieved from http://tree.bio.ed.ac.uk/software/figtree/

Ratnasingham, S., \& Hebert, P. D. (2007). BOLD: The barcode of life data system (http://www.barcodinglife.org). Molecular Ecology Notes, 7(3), 355-364.

Raychoudhury, R., Baldo, L., Oliveira, D. C., \& Werren, J. H. (2009). Modes of acquisition of Wolbachia: Horizontal transfer, hybrid introgression, and codivergence in the Nasonia species complex. Evolution, 63(1), 165-183.

Rhoades, P., Griswold, T., Waits, L., Bosque-Pérez, N. A., Kennedy, C. M., \& Eigenbrode, S. D. (2017). Sampling technique affects detection of habitat factors influencing wild bee communities. Journal of Insect Conservation, 21(4), 703-714. https://doi.org/10.1007/s1084 1-017-0013-0

Riley, J. R., Smith, A., \& Reynolds, D. (2003). The feasibility of using vertical-looking radar to monitor the migration of brown planthopper and other insect pests of rice in China. Insect Science, 10(1), 1-19. https://doi.org/10.1111/j.1744-7917.2003.tb00359.x

Ronquist, F., Teslenko, M., van der Mark, P., Ayres, D. L., Darling, A., Hohna, S., \& Huelsenbeck, J. P. (2012). MrBayes 3.2: Efficient Bayesian phylogenetic inference and model choice across a large model space. Systematic Biology, 61(3), 539-542.

Rowley, D. L., Coddington, J. A., Gates, M. W., Norrbom, A. L., Ochoa, R. A., Vandenberg, N. J., \& Greenstone, M. H. (2007). Vouchering DNA-barcoded specimens: Test of a nondestructive extraction protocol for terrestrial arthropods. Molecular Ecology Notes, 7(6), 915-924. https://doi.org/10.1111/j.1471-8286.2007.01905.x
Russell, J. A., Funaro, C. F., Giraldo, Y. M., Goldman-Huertas, B., Suh, D., Kronauer, D. J. C., Moreau, C. S., \& Pierce, N. E. (2012). A veritable menagerie of heritable bacteria from ants, butterflies, and beyond: Broad molecular surveys and a systematic review. PLoS One, 7(12), e51027. https://doi.org/10.1371/journal.pone.0051027

Russell, J. A., Goldman-Huertas, B., Moreau, C. S., Baldo, L., Stahlhut, J. K., Werren, J. H., \& Pierce, N. E. (2009). Specialization and geographic isolation among Wolbachia symbionts from ants and lycaenid butterflies. Evolution: International Journal of Organic Evolution, 63(3), 624-640.

Sabu, T. K., \& Shiju, R. T. (2010). Efficacy of pitfall trapping, Winkler and Berlese extraction methods for measuring ground-dwelling arthropods in moist deciduous forests in the Western Ghats. Journal of Insect Science, 10(1), 98. https://doi.org/10.1673/031.010.9801

Salunke, B. K., Salunkhe, R. C., Dhotre, D. P., Walujkar, S. A., Khandagale, A. B., Chaudhari, R., Chandode, R. K., Ghate, H. V., Patole, M. S., Werren, J. H., \& Shouche, Y. S. (2012). Determination of Wolbachia diversity in butterflies from Western Ghats, India, by a multigene approach. Applied and Environment Microbiology, 78(12), 44584467. https://doi.org/10.1128/AEM.07298-11

Sanaei, E., Charlat, S., \& Engelstädter, J. (2021). Wolbachia host shifts: Routes, mechanisms, constraints and evolutionary consequences. Biological Reviews, 96(2), 433-453.

Semiatizki, A., Weiss, B., Bagim, S., Rohkin-Shalom, S., Kaltenpoth, M., \& Chiel, E. (2020). Effects, interactions, and localization of Rickettsia and Wolbachia in the house fly parasitoid, Spalangia endius. Microbial Ecology, 80(3), 718-728. https://doi.org/10.1007/s00248-02001520-x

Sintupachee, S., Milne, J., Poonchaisri, S., Baimai, V., \& Kittayapong, P. (2006). Closely related Wolbachia strains within the pumpkin arthropod community and the potential for horizontal transmission via the plant. Microbial Ecology, 51(3), 294-301. https://doi. org/10.1007/s00248-006-9036-x

Smith, E. P., \& van Belle, G. (1984). Nonparametric estimation of species richness. Biometrics, 119-129. https://doi.org/10.2307/2530750

Smith, J. M. (1992). Analyzing the mosaic structure of genes. Journal of Molecular Evolution, 34(2), 126-129. https://doi.org/10.1007/ BF00182389

Stahlhut, J. K., Desjardins, C. A., Clark, M. E., Baldo, L., Russell, J. A., Werren, J. H., \& Jaenike, J. (2010). The mushroom habitat as an ecological arena for global exchange of Wolbachia. Molecular Ecology, 19(9), 1940-1952.

Stireman, J. O., \& Singer, M. S. (2003). What determines host range in parasitoids? An analysis of a tachinid parasitoid community. Oecologia, 135(4), 629-638. https://doi.org/10.1007/s00442-003-1235-2

Stouthamer, C. M., Kelly, S. E., Mann, E., Schmitz-Esser, S., \& Hunter, M. S. (2019). Development of a multi-locus sequence typing system helps reveal the evolution of Cardinium hertigii, a reproductive manipulator symbiont of insects. BMC Microbiology, 19(1), 1-13. https://doi. org/10.1186/s12866-019-1638-9

Tolley, S. J., Nonacs, P., \& Sapountzis, P. (2019). Wolbachia horizontal transmission events in ants: What do we know and what can we learn? Frontiers in Microbiology, 10, 296. https://doi.org/10.3389/ fmicb.2019.00296

Turelli, M., Cooper, B. S., Richardson, K. M., Ginsberg, P. S., Peckenpaugh, B., Antelope, C. X., Kim, K. J., May, M. R., Abrieux, A., Wilson, D. A., Bronski, M. J., Moore, B. R., Gao, J.-J., Eisen, M. B., Chiu, J. C., Conner, W. R., \& Hoffmann, A. A. (2018). Rapid global spread of wRi-like Wolbachia across multiple Drosophila. Current Biology, 28(6), 963-971. https://doi.org/10.1016/j.cub.2018.02.015

Vavre, F., Fleury, F., Lepetit, D., Fouillet, P., \& Bouletreau, M. (1999). Phylogenetic evidence for horizontal transmission of Wolbachia in host-parasitoid associations. Molecular Biology and Evolution, 16(12), 1711-1723. https://doi.org/10.1093/oxfordjournals.molbev.a026084 
Vos, M., \& Didelot, X. (2009). A comparison of homologous recombination rates in bacteria and archaea. The ISME Journal, 3(2), 199-208. https://doi.org/10.1038/ismej.2008.93

Vrijenhoek, R. (1994). DNA primers for amplification of mitochondrial cytochrome c oxidase subunit I from diverse metazoan invertebrates. Molecular Marine Biology and Biotechnology, 3(5), 294-299.

Wang, X., Xiong, X., Cao, W., Zhang, C., Werren, J. H., \& Wang, X. (2020). Phylogenomic analysis of Wolbachia strains reveals patterns of genome evolution and recombination. Genome Biology and Evolution, 12(12), 2508-2520.

Weinert, L. A., Araujo-Jnr, E. V., Ahmed, M. Z., \& Welch, J. J. (2015). The incidence of bacterial endosymbionts in terrestrial arthropods. Proceedings of the Royal Society B: Biological Sciences, 282(1807), 20150249. https://doi.org/10.1098/rspb.2015.0249

Werren, J. H., Baldo, L., \& Clark, M. E. (2008). Wolbachia: Master manipulators of invertebrate biology. Nature Reviews Microbiology, 6(10), 741-751. https://doi.org/10.1038/nrmicro1969

Werren, J. H., \& Bartos, J. D. (2001). Recombination in Wolbachia. Current Biology, 11(6), 431-435. https://doi.org/10.1016/S0960 -9822(01)00101-4

Werren, J. H., \& Windsor, D. M. (2000). Wolbachia infection frequencies in insects: Evidence of a global equilibrium? Proceedings of the Royal Society of London. Series B: Biological Sciences, 267(1450), 1277-1285.

Werren, J. H., Windsor, D., \& Guo, L. R. (1995). Distribution of Wolbachia among neotropical arthropods. Proceedings of the Royal Society of London. Series B: Biological Sciences, 262(1364), 197-204.

Werren, J. H., Zhang, W., \& Guo, L. R. (1995). Evolution and phylogeny of Wolbachia: Reproductive parasites of arthropods. Proceedings of the Royal Society B: Biological Sciences, 261(1360), 55-63.

Yang, X. H., Zhu, D. H., Liu, Z., Zhao, L., \& Su, C. Y. (2013). High levels of multiple infections, recombination and horizontal transmission of Wolbachia in the Andricus mukaigawae (Hymenoptera; Cynipidae) communities. PLoS One, 8(11), e78970. https://doi.org/10.1371/ journal.pone.0078970

Zchori-Fein, E., \& Perlman, S. J. (2004). Distribution of the bacterial symbiont Cardinium in arthropods. Molecular Ecology, 13(7), 20092016. https://doi.org/10.1111/j.1365-294X.2004.02203.x

Zélé, F., Santos, I., Olivieri, I., Weill, M., Duron, O., \& Magalhães, S. (2018). Endosymbiont diversity and prevalence in herbivorous spider mite populations in South-Western Europe. FEMS Microbiology Ecology, 94(4), fiy015. https://doi.org/10.1093/femsec/fiy015

Zettler, J. A., Kohler, B., Ness, T., Buske, H., \& Lipat, A. J. (2020). Crosscontamination is a risk associated with short-term insect specimen storage. The Canadian Entomologist, 153(3), 368-373. https://doi. org/10.4039/tce.2020.64

Zhang, K. J., Han, X., \& Hong, X. Y. (2013). Various infection status and molecular evidence for horizontal transmission and recombination of Wolbachia and Cardinium among rice planthoppers and related species. Insect Science, 20(3), 329-344.

Zhang, Y.-K., Chen, Y.-T., Yang, K., Qiao, G.-X., \& Hong, X.-Y. (2016). Screening of spider mites (Acari: Tetranychidae) for reproductive endosymbionts reveals links between co-infection and evolutionary history. Scientific Reports, 6(1), 1-9. https://doi.org/10.1038/ srep27900

Zhao, D. X., Chen, D. S., Ge, C., Gotoh, T., \& Hong, X. Y. (2013). Multiple infections with Cardinium and two strains of Wolbachia in the spider mite Tetranychus phaselus Ehara: Revealing new forces driving the spread of Wolbachia. PLoS One, 8(1), e54964. https://doi. org/10.1371/journal.pone.0054964

\section{SUPPORTING INFORMATION}

Additional supporting information may be found online in the Supporting Information section.

How to cite this article: Gupta, M., Kaur, R., Gupta, A., \& Raychoudhury, R. (2021). Are ecological communities the seat of endosymbiont horizontal transfer and diversification? A case study with soil arthropod community. Ecology and Evolution, 11, 14490-14508. https://doi.org/10.1002/ ece3.8108 